\title{
Identification of low order manifolds: Validating the algorithm of Maas and Pope
}

\author{
Carl Rhodes ${ }^{\text {a) }}$ \\ Chemical Engineering 210-41, California Institute of Technology, Pasadena, California 91125 \\ Manfred Morari \\ Institut für Automatik, ETH-Z/ETL, CH-8092 Zürich, Switzerland \\ Stephen Wiggins \\ Control and Dynamical Systems 107-81, California Institute of Technology, Pasadena, California 91125
}

(Received 28 July 1998; accepted for publication 30 December 1998)

\begin{abstract}
The algorithm of Maas and Pope (1992) is presented as a method for identification of invariant reduced-order manifolds for stable systems which exhibit dynamics with a time-scale separation. While this method has been published previously in the literature, theoretical justification for the algorithm was not presented in the original work. Here, it will be shown rigorously that the algorithm correctly identifies the slow manifold. Before the theoretical results are presented, a brief background on the behavior of singularly perturbed systems is presented. The algorithm of Maas and Pope (1992) is then introduced. This method will be applied to two different examples, a distillation column and a two-phase chemical reactor. For each of these examples, the resulting reduced-order description will be compared to other standard methods of producing reduced-order models. In addition, some preliminary thoughts on how this method can be used to form reduced-order models are presented. (C) 1999 American Institute of Physics.
\end{abstract}

[S1054-1500(99)01701-2]

\begin{abstract}
Dynamic models of chemical processes that are derived from first-principles tend to be of high order, yet may exhibit behavior consistent with low-order systems. Often, this characteristic behavior is the result of dynamics occurring on different time scales. The algorithm of Maas and Pope (1992) was developed as a method of identifying the invariant manifold of "slow" dynamics for this class of systems. Here it is shown rigorously for the first time that the algorithm accurately identifies the slow manifold for systems exhibiting both infinite and finite time-scale separations. Some thoughts on how the results of this algorithm can be used for forming reduced models are introduced and examples are presented.
\end{abstract}

\section{INTRODUCTION}

Recently, there have been significant advances in the field of nonlinear control. In order to apply these methods to real world processes, a suitable mathematical model of a system to be controlled must be developed. Current methods of nonlinear control are unable to deal with process models that have a high dimensional state-space description. Controller synthesis using methods based on differential geometry can become unwieldy due to algebraic manipulations needed for computing the controller. If model predictive control methods are being used, computational requirements may be large for system descriptions of even moderate dimension. Another alternative is to use methods of control based on singularly perturbed systems. Kokotovic and Khalil

a) Current address: The RAND Corporation, 1700 Main Street, P.O. Box 2138, Santa Monica, CA 90407.
(1986) contains a good collection of past work in this field. However, such methods rely on a description of the system dynamics in a specific format that may not always be straightforward to obtain.

Dynamical descriptions of chemical processes are often derived from first-principles models of the chemistry and physics of the system. These models are developed by writing detailed descriptions of the reaction kinetics, thermodynamics, heat transfer, material and mass balances. Models developed in this way are surprisingly accurate but are typically of fairly high order and complexity. At the same time, these models generally exhibit behavior on widely differing time scales. If one is uninterested with behavior occurring on fast time scales, it is natural to consider a model reduction scheme which discards the fast dynamics of the model. Heuristically, models can be simplified by making pseudo steady-state or equilibrium assumptions. However, these assumptions are not mathematically rigorous and may not always result in an accurate model.

In this work, systems which exhibit large time-scale separations are considered. For systems with stable dynamics and large time-scale separations, the dynamics converges quickly to a reduced-order invariant manifold in the statespace. Once on this manifold, the system remains there and only the slower dynamics of the system persists. Since the majority of the system dynamics take place on this manifold, it seems natural that any model reduction scheme should find the location of this reduced manifold. Since there is no fast dynamics on this manifold it will be called the slow manifold. The next section is a short introduction to the theory of singular perturbations. 


\section{A. Singular perturbation theory}

Singularly perturbed systems are systems of ordinary differential equations (ODEs) which exhibit dynamic behavior evolving over two vastly different time scales. The standard form of the singular perturbation model consists of a model in which the derivatives of a number of the variables are multiplied by a small parameter, $\epsilon$.

$$
x^{\prime}=f(x, y, \epsilon), \quad \epsilon y^{\prime}=g(x, y, \epsilon),
$$

where ' $=d / d t, x \in \mathfrak{R}^{l}, y \in \mathfrak{R}^{m}$, and $\epsilon \in \mathfrak{R}$. When $\epsilon$ is small, this system of ODEs will exhibit a characteristic two-timescale behavior. When $\epsilon \rightarrow 0$, system (1) becomes

$$
x^{\prime}=f(x, y, 0), \quad 0=g(x, y, 0) .
$$

In this limit, $g(x, y, 0)=0$ defines an invariant region upon which the dynamics $x^{\prime}=f(x, y, 0)$ takes place. Outside of this region, the dynamics is not defined, as the condition $g(x, y, 0)=0$ cannot be met.

By scaling time such that $\tau \epsilon=t$, system (1) becomes

$$
\dot{x}=\epsilon f(x, y, \epsilon), \quad \dot{y}=g(x, y, \epsilon),
$$

where ${ }^{\circ}=d / d \tau$. For $\epsilon \neq 0$, system (3) is equivalent to system (1). However, the behavior is quite different when $\epsilon \rightarrow 0$. In this limit, system (3) becomes

$$
\dot{x}=0, \quad \dot{y}=g(x, y, 0) .
$$

Notice that for system (4), $x$ is constant and only the value of $y$ changes with time.

Because of the scaling of time with $\epsilon$, system (1) is called the slow system and system (3) is called the fast system. When $\epsilon=0$, the set in the state space for which slow dynamics is defined, $g(x, y, 0)=0$, is the set of critical points for the fast system. In the two limiting cases, analysis of the dynamics is simplified by the fact that the state-space dynamics is limited to a manifold which is smaller than the original state-space. For process control, the more compelling limiting case is the slow system. Since the system trajectories quickly converge to the reduced-order manifold defined by $g(x, y, 0)=0$ when the fast dynamics is stable, control may not be needed for the fast dynamics. In order to improve performance in the region of slow dynamics, only a description of these slow dynamics may be needed.

Unfortunately, many physical systems which exhibit behavior consistent with singularly perturbed systems do not take the standard form of system (1) after first-principles modeling. In order to convert these systems into the standard form, transformations based on physical insight into the specific system can be used (Khahil, 1996). Often these transformations are not obvious, as choosing a suitable "small" parameter can be difficult. Converting a system into the "optimal" form such that the slow and fast dynamics are completely decoupled may not even be possible using physical insight. For this reason, it would be preferable to identify the slow manifold from the complete description of the system dynamics using a computational algorithm. Other research in this area has considered this problem.

Modal reduction is one example of previous work in model reduction for linear systems exhibiting multiple time- scale behavior. The eigenvalues of the system can be found, and a transformation which splits the state-space into slow and fast modes can be used to discard the fast dynamics of the system [this method is described in Brogan (1991)]. For nonlinear systems, the model reduction scheme of Roussel and Fraser (1991) can be used to develop a low-order approximation of systems which exhibit nonlinear dynamics. However, the algorithm becomes computationally intractable for systems of even moderate dimension and complexity. For this reason, other methods of model reduction are needed for nonlinear systems.

\section{THE MAAS AND POPE ALGORITHM}

The algorithm described in the paper of Maas and Pope (1992) was developed to identify slow manifolds in problems associated with combustion. The algorithm computes discrete points located on the slow manifold from a dynamical description of a system which consists of ODEs which exhibit time-scale separation. The motivation for this algorithm is the fact that simulations of complete models of detailed combustion kinetics can take hundreds of hours of supercomputer time. By identifying the manifold of slow dynamics, it is hoped that methods can be developed for producing a reduced model of the system in order to decrease the computational time needed for accurate simulations.

For the purpose of describing the Maas and Pope algorithm, assume the following system, which exhibits timescale separation, is given

$$
\dot{z}=F(z),
$$

with $z \in \mathfrak{R}^{n}$. From this description, an $n_{r}<n$ dimensional manifold where the slow dynamics exists is to be found. The dynamics of the overall system from arbitrary initial conditions should decay very quickly onto this $n_{r}$-dimensional invariant slow manifold. While the original algorithm does not develop a reduced model of the dynamics on the slow manifold, it does identify individual points on the slow manifold.

The idea behind the algorithm presented in the paper of Maas and Pope is based mainly on local arguments, and a rigorous justification of the algorithm is not presented in the original paper. In the paper it is stated that "While the development is mathematical, no attempt at rigor is made" and the analysis of the algorithm is given only for linearized dynamics. The algorithm makes use of the eigenvalue decomposition of the Jacobian of the system dynamics. Take the Jacobian of $F$ evaluated at $z_{0}$ defined as

$$
J=\frac{\partial F}{\partial z}\left(z_{0}\right) .
$$

It is stated that the eigenvalues of $J$ identify the time scales associated with movement in the state-space when there is a clear separation in time scales for the system. In addition, the eigenvectors associated with these eigenvalues describe the "characteristic directions" associated with these time scales. Such arguments should only hold in the neighborhood of the reduced manifold.

The goal of the Maas and Pope (1992) algorithm is to identify the set in the state-space where the projection of the 
dynamics onto the directions consisting of only fast linearized dynamics is zero. On this surface, the dynamics of the fastest time scales should be at equilibrium. The manifold is found in the following manner. Suppose a local basis is formed by the eigenvectors of the Jacobian at a point $z$. Those eigenvectors corresponding to distinct eigenvalues form basis members. In the case of $m$ repeated eigenvalues, then $m$ linearly independent chosen eigenvectors should be selected to be members of the basis. When the eigenvectors are sorted such that the absolute value of the real part of the corresponding eigenvalues is decreasing, the result is

$$
V(z)=\left(\begin{array}{cccc}
\mid & \mid & & \mid \\
v_{1} & v_{2} & \cdots & v_{n} \\
\mid & \mid & & \mid
\end{array}\right) .
$$

The inverse of this matrix is

$$
V^{-1}(z)=\left(\begin{array}{ccc}
- & \tilde{v}_{1} & - \\
- & \tilde{v}_{2} & - \\
\vdots & \\
- & \tilde{v}_{n} & -
\end{array}\right)
$$

The inverse also defines the coordinate transformation from the original coordinates to a basis consisting of the eigenvectors. On the low-dimensional manifold of slow dynamics the following relationship holds:

$$
W(z) F(z)=0,
$$

where

$$
W(z)=\left(\begin{array}{ccc}
- & \tilde{v}_{n_{r}+1} & - \\
- & \tilde{v}_{n_{r}+2} & - \\
\vdots & \\
- & \tilde{v}_{n} & -
\end{array}\right)
$$

Note that $W$ is a function of $z$, and the Jacobian of $F$ (specifically its eigenvalue-eigenvector decomposition) must be evaluated at each point of interest so that $W$ can be found. The matrix $W$ projects the dynamics onto the eigenvectors corresponding to the $n-n_{r}$ smallest eigenvalues of the linearized dynamics.

Since the eigenvectors are not orthogonal, the matrix $W(z)$ may be ill-conditioned and the inversion may be problematic numerically. The solution to the above problem is better behaved computationally if the Schur basis is used to define the spaces referring to the slow and fast eigenvalues. The Schur basis is orthogonal, and the computations associated with it are better behaved numerically. The Schur decomposition is defined as follows [Horn and Johnson (1985)]:

$$
J=Q N Q^{T},
$$

where $Q$ is an orthonormal matrix and $N$ is a triangular matrix which is sorted such that the eigenvalues appearing along the diagonal are sorted in order of descending magnitude of the real part. If $Q$ is

$$
Q=\left(\begin{array}{cccc}
\mid & \mid & & \mid \\
q_{1} & q_{2} & \cdots & q_{n} \\
\mid & \mid & & \mid
\end{array}\right),
$$

then it can be easily seen that $q_{1}=v_{1}$. Because of the triangular form of the matrix $N$, it can also be shown that $v_{2}$ can be formed from a linear combination of $q_{1}$ and $q_{2}$. By extending this argument, the $n_{r}$ eigenvectors corresponding to the $n_{r}$ largest eigenvalues will be spanned by the basis $\left(q_{1}, \ldots, q_{n_{r}}\right)$ and the orthogonal complement to this space is spanned by the basis $\left(q_{n_{r+1}}, \ldots, q_{n}\right)$. Because of this, the slow manifold is defined by points $z$ such that

$$
Q_{L}^{T}(z) F(z)=\left(\begin{array}{ccc}
- & q_{n_{r}+1}^{T} & - \\
- & q_{n_{r}+2}^{T} & - \\
\vdots & \\
& q_{n}^{T} & -
\end{array}\right) F(z)=0 .
$$

In the original work, both Newton's method and continuation methods are suggested for finding the manifold where $Q_{L}^{T}(z) F(z)=0$. Since any fixed point $z_{f}$ will be on the manifold, as $F\left(z_{f}\right)=0$ for a fixed point by definition, a fixed point can be identified and used as a starting point for the algorithm. In order to make the equations consistent, $n_{r}$ additional parametric equations must be defined. An example would be fixing $n_{r}$ of the variables to some given values by solving the extended set of equations

$$
\left(\begin{array}{c}
Q_{L}^{T}(z) F(z) \\
P(z, \tau)
\end{array}\right)=0
$$

where

$$
P(z, \tau)=z_{n_{i}}-\tau_{i} \quad i=1, \ldots, r,
$$

where $\tau$ is a vector of fixed parameters. The solution of Eq. (14) for $z$ gives a single point on the slow manifold. By solving this equation repeatedly with different values of the parameter vector $\tau$, numerous points on the manifold can be computed which are arbitrarily close together by the choice of parameters $\tau$.

In this work, a nonlinear optimization is used to identify the slow manifold. It is not clear what advantages this method offers over other computational approaches (continuation, one-parameter cuts, etc.), as alternative approaches to solving the problem were not explored. An interface has been written to the program NPSOL (Gill et al., 1994) such that the following problem is solved:

$$
\min _{z \in \Re^{n}}\left\|Q_{L}^{T}(z) F(z)\right\|_{2}=\sum_{i=1}^{n-n_{r}} q_{i+n_{r}}^{T} F(z),
$$

with $n_{r}$ of the states of the system fixed

$$
z_{n_{i}}=\tau_{i} \quad i=1, \ldots, r
$$


Note that $\tau_{i}$ behave like constraints on some of the state variables to make the minimization solution unique. The choice of $n_{i}$ determines how the slow manifold should be parametrized. If the objective function is equal to zero after the minimization at a single point $z$, the condition for identifying the slow manifold has been met. By repeating the algorithm for different values of the vector $\tau$, a number of different points on the manifold can be calculated be repeating the process. If the minimum of the objective function is nonzero, no point on the reduced manifold exists with the defined values of the fixed states.

One question which is not answered by this process is the proper choice of the dimension of the reduced manifold, $n_{r}$. It is important that $n_{r}$ should be chosen such that there is a "gap" in the time scales between the dynamics that are assumed to be the fast and slow dynamics; however, a number of values of $n_{r}$ may meet this criterion. One option would be to choose $n_{r}$ such that the accuracy of the model is suitable for the desired application. Clearly, more research is needed in this area.

\section{THE FENICHEL NORMAL FORM}

The transformation to the Fenichel normal form will be used to prove that the Maas and Pope algorithm identifies the intrinsic slow manifold in the case where $\epsilon \neq 0$. The derivation of this normal form is given here as background information. It should be noted that the material in this section comes from the papers of Jones (1994) and Fenichel (1979).

When $\epsilon \rightarrow 0$ there is an infinite time-scale separation between the fast and slow dynamics. In this case the set of critical points of (4) is defined by $g(x, y, 0)=0$. This is the set where the slow dynamics defined by (2) exist. The manifold where $g(x, y, 0)=0$ is defined by a set of $m$ equations in $\mathfrak{R}^{l+m}$. This manifold is expected to be $l$ dimensional (at least locally) by the preimage theorem of differential geometry (Guillemin and Pollack, 1974) and it is possible that the manifold has boundary. A sufficient condition for the preimage theorem to hold is that $\operatorname{det}((\partial g / \partial y)(x, y, 0)) \neq 0$. The assumption of normal hyperbolicity, which will follow shortly, will be sufficient for the preimage theorem to apply. In this paper, the manifold of critical points will be called $\mathcal{M}_{0}=\{(x, y): g(x, y, 0)=0\}$, where the subscript refers to the fact that $\epsilon=0$.

Before presenting the transformation to the Fenichel normal form, a list of assumptions which will be utilized throughout this article is presented. The first assumption concerns the smoothness of the functions $f$ and $g$. This assumption ensures that the transformation to the Fenichel normal form is smooth.

Assumption 3.1. The functions $f$ and $g$ are assumed to be $C^{\infty}$ on the set $U \times I, U \in \mathfrak{R}^{l+m}$ and the interval I contains 0 .

It is possible to weaken this assumption to $C^{r}$ smoothness, but $C^{\infty}$ smoothness will be assumed to simplify the proofs that follow.

The second assumption concerns the dynamics on the manifold $\mathcal{M}_{0}$. Since the manifold is a set of critical points, directions normal to $\mathcal{M}_{0}$ should correspond to eigenvalues which are nonzero and eigenvalues tangent to $\mathcal{M}_{0}$ should be identically zero.

Definition 3.1. The manifold $\mathcal{M}_{0}$ is normally hyperbolic if the linearization of (4) has exactly $l$ eigenvalues on the imaginary axis $\operatorname{Re}(\lambda)=0$ for all $(x, y) \in \mathcal{M}_{0}$.

Assumption 3.2. The manifold $\mathcal{M}_{0}$ is normally hyperbolic.

Since the linearization of Eq. (4) is

$$
\left(\begin{array}{c}
\dot{\delta x} \\
\overline{\delta y}
\end{array}\right)=\left(\begin{array}{cc}
0 & 0 \\
\frac{\partial g}{\partial x}(x, y, 0) & \frac{\partial g}{\partial y}(x, y, 0)
\end{array}\right)\left(\begin{array}{l}
\delta x \\
\delta y
\end{array}\right),
$$

$\mathcal{M}_{0}$ is normally hyperbolic whenever $\partial g / \partial y(x, y, 0)$ has no eigenvalues on the imaginary axis for $(x, y) \in \mathcal{M}_{0}$.

Since $\mathcal{M}_{0}$ may have boundary, the manifold cannot be invariant in general since trajectories can escape the manifold at the boundary. However, it can be shown that $\mathcal{M}_{0}$ will be locally invariant. A manifold is locally invariant when the only way that a trajectory can escape the manifold is through the boundary.

In order to simplify the notation, it will be assumed that the manifold $\mathcal{M}_{0}$ can be represented as the graph of a function $h$. Since the matrix $(\partial g / \partial y)(x, y, 0)$ is nonsingular on the manifold due to the assumption of normal hyperbolicity, it is locally invertible for any $(x, y) \in \mathcal{M}_{0}$. By the Implicit Function Theorem, $y$ can always be found as a function of $x$ locally. Assuming that a solution can be made globally, then $\mathcal{M}_{0}$ can be represented by a graph of a function $h^{0}$.

Assumption 3.3. $\mathcal{M}_{0}$ can be represented as a graph $\mathcal{M}_{0}=\left\{(x, y): y=h^{0}(x)\right\}$ for some compact domain $x \in K$ where $K \subset \mathfrak{R}^{l}$.

As $y$ can be found from $x$ locally, the main assumption needed here is that the function $h^{0}(x)$ can be pieced together globally from the local descriptions.

Some other structures of the unperturbed equations ( $\epsilon$ $=0$ ) should also be defined here. First, it will be assumed without loss of generality that $h^{0}(x)=0$ for all points $x \in K$ where the set $K$ is simply the compact domain $\left(x, h^{0}(x)\right)$ $\in \mathcal{M}_{0}$. If $h^{0}(x) \neq 0$ in the original coordinates, the change of coordinates from $y$ to $\tilde{y}=y-h^{0}(x)$ gives the desired property. Using this assumption, Eq. (4) can be converted to the following form in the neighborhood of $\mathcal{M}_{0}$ :

$$
\dot{x}=0, \quad \dot{y}=L(x) y+G(x, y, 0),
$$

where $L(x)=(\partial g / \partial y)\left(x, h^{0}(x), 0\right)$ and $|G(x, y, 0)| \leqslant \gamma(|y|)$ as $L(x) y$ is linear in $y$.

Using linear algebra, a transformation can be found such that $y$ is transformed into coordinates $(a, b)$,

$$
\begin{aligned}
& \dot{x}=0, \\
& \dot{a}=A(x) a+G_{1}(x, a, b, 0), \\
& \dot{b}=B(x) b+G_{2}(x, a, b, 0),
\end{aligned}
$$

where the eigenvalues of $A(x)$ are strictly positive and the eigenvalues of $B(x)$ are strictly negative. The eigenvalues of 

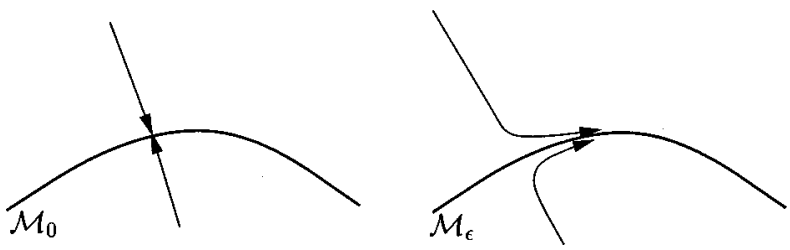

FIG. 1. Behavior of trajectories in the neighborhood near the slow manifold for $\mathcal{M}_{0}$ and $\mathcal{M}_{\epsilon}$

$L(x)$ are bounded away from zero by the assumption that $\mathcal{M}_{0}$ is hyperbolic. It will also be assumed that $a \in \mathfrak{R}^{m} a$ and $b \in \mathfrak{R}^{m_{b}}$ where $m_{a}+m_{b}=m$.

Each point $(x, 0) \in \mathcal{M}_{0}$ has a stable and unstable manifold associated with it. The $m_{b}$-dimensional stable manifold at $x$ will be called $W^{s}(x)$ and is defined by the condition $a$ $=0$. The $m_{a}$-dimensional unstable manifold at $x, W^{u}(x)$ is defined by $b=0 . W^{s}\left(\mathcal{M}_{0}\right)$ and $W^{u}\left(\mathcal{M}_{0}\right)$ are simply the stable and unstable manifolds for all points $x \in \mathcal{M}_{0}$.

In the case where the time-scale separation between the variables is finite, the parameter $\epsilon$ is small but nonzero. When $\epsilon$ is sufficiently small, a manifold of slow dynamics which is close to $\mathcal{M}_{0}$ still exists. It is shown by Fenichel (1979) that there exists a manifold called $\mathcal{M}_{\epsilon}$ that is diffeomorphic to $\mathcal{M}_{0}$.

Theorem 3.1 [Fenichel (1979)]: If $\epsilon>0$ and sufficiently small, there exist manifolds $\mathcal{M}_{\epsilon}, W^{s}\left(\mathcal{M}_{\epsilon}\right)$, and $W^{u}\left(\mathcal{M}_{\epsilon}\right)$ which are $\mathcal{O}(\epsilon)$ perturbations and are diffeomorphic to $\mathcal{M}_{0}$, $W^{s}\left(\mathcal{M}_{0}\right)$, and $W^{u}\left(\mathcal{M}_{0}\right)$, respectively. In addition, $\mathcal{M}_{\epsilon}$ is invariant to the flow of (3).

If $\epsilon>0$ is sufficiently small, for some $\Delta>0$

(1) there is a function $\left[\begin{array}{l}a \\ b\end{array}\right]=h^{\epsilon}(x)$ defined for $x \in K,|a|$ $\leqslant \Delta$, and $|b| \leqslant \Delta$ such that the graph

$$
\mathcal{M}_{\epsilon}(x)=\left\{(x, a, b):\left[\begin{array}{l}
a \\
b
\end{array}\right]=h^{\epsilon}(x)\right\}
$$

is locally invariant under the dynamics defined by (3);

(2) there is a function $a=h_{s}(x, b, \epsilon)$ defined for $x \in K$ and $|b| \leqslant \Delta$ such that the graph

$$
W^{s}\left(\mathcal{M}_{\epsilon}\right)=\left\{(x, a, b): a=h_{s}(x, b, \epsilon)\right\}
$$

is locally invariant under the dynamics defined by (3);

(3) there is a function $b=h_{u}(x, a, \epsilon)$ defined for $x \in K$ and $|a| \leqslant \Delta$ such that the graph

$$
W^{u}\left(\mathcal{M}_{\epsilon}\right)=\left\{(x, a, b): b=h_{u}(x, a, \epsilon)\right\}
$$

is locally invariant under the dynamics defined by (3).

A proof of this theorem is found in Fenichel (1979) and Jones (1994).

Now in the fast scaling, the manifold $\mathcal{M}_{\epsilon}$ will not need to be a manifold of stationary points as was the case for $\mathcal{M}_{0}$. Figure 1 illustrates the behavior of trajectories in the neighborhood of the slow manifold in these two different cases.

The Fenichel normal form is a transformation which converts equations from the general form of (3) to one in which the variables are "decoupled" in some sense. The equations will be transformed as before, but this time the parameter $\epsilon$ is allowed to be nonzero. As previously, Eq. (3) can be converted to the following form on $\mathcal{M}_{0}$ :

$$
\dot{x}=\epsilon f(x, y, \epsilon), \quad \dot{y}=L(x) y+G(x, y, \epsilon),
$$

where $L(x)=(\partial g / \partial y)\left(x, h^{0}(x), \epsilon\right) \quad$ and $\quad|G(x, y, \epsilon)|$ $\leqslant \gamma(|y|+|\epsilon|)$ since $G$ is higher order in $y$ and $\epsilon$.

Again transforming $y$ to $(a, b)$,

$$
\begin{aligned}
& \dot{x}=\epsilon f(x, y, \epsilon), \\
& \dot{a}=A(x) a+G_{1}(x, a, b, \epsilon), \\
& \dot{b}=B(x) b+G_{2}(x, a, b, \epsilon),
\end{aligned}
$$

where the eigenvalues of $A(x)$ are strictly positive and the eigenvalues of $B(x)$ are strictly negative, $a \in \mathfrak{R}^{m} a$ and $b$ $\in \mathfrak{R}^{m_{b}}$, and $m_{a}+m_{b}=m$.

Since a graph exists which defines the manifolds $W^{s}\left(\mathcal{M}_{\epsilon}\right)$ and $W^{u}\left(\mathcal{M}_{\epsilon}\right)$, the coordinates can be transformed to simplify the dynamics. In the first step, the coordinates are transformed to

$$
\begin{aligned}
& x_{1}=x, \\
& a_{1}=a-h_{s}(x, b, \epsilon), \\
& b_{1}=b,
\end{aligned}
$$

which causes the manifold $W^{s}\left(\mathcal{M}_{\epsilon}\right)$ to be defined by $a_{1}$ $=0$. The coordinates can be transformed again to

$$
\begin{aligned}
& x_{2}=x_{1}, \\
& a_{2}=a_{1}, \\
& b_{2}=b_{1}-h_{u}\left(x_{1}, a_{1}+h_{s}\left(x_{1}, b_{1}, \epsilon\right), \epsilon\right),
\end{aligned}
$$

where the surface $b_{2}=0$ defines the manifold $W^{u}\left(\mathcal{M}_{\epsilon}\right)$.

Since the stable and unstable manifolds are invariant under the dynamics defined by (25), the surfaces $a_{2}=0$ and $b_{2}=0$ must also be invariant. This means that $a_{2}=0$ implies $\dot{a}_{2}=0$ and $b_{2}=0$ implies $\dot{b}_{2}=0$ and the equations describing the dynamics in these coordinates should take the form

$$
\begin{aligned}
& \dot{x}_{2}=\epsilon F_{2}\left(x_{2}, a_{2}, b_{2}, \epsilon\right) \\
& \dot{a}_{2}=\Lambda\left(x_{2}, a_{2}, b_{2}, \epsilon\right) a_{2}, \\
& \dot{b}_{2}=\Gamma\left(x_{2}, a_{2}, b_{2}, \epsilon\right) b_{2},
\end{aligned}
$$

locally, where $F_{2}\left(x_{2}, a_{2}, b_{2}, \epsilon\right)=f\left(x, a-h_{s}(x, b, \epsilon), \quad b\right.$ $\left.-h_{u}(x, a, \epsilon)\right)$ and $\Lambda\left(x_{2}, a_{2}, b_{2}, \epsilon\right)$ and $\Gamma\left(x_{2}, a_{2}, b_{2}, \epsilon\right)$ are matrices. Additionally, system (28) should be equivalent to (25) when $\epsilon=0$. For this reason $\Lambda\left(x_{2}, 0,0,0\right)=A(x)$ and $\Gamma\left(x_{2}, 0,0,0\right)=B(x)$.

With this transformation, the coordinates defining the stable and unstable manifold of a given point $v_{0} \in \mathcal{M}_{0}$ have been "straightened." In order to decouple the slow directions, theory which is known as Fenichel fibering is needed. Since it has been shown that the manifolds $W^{s}\left(\mathcal{M}_{0}\right)$ and $W^{u}\left(\mathcal{M}_{0}\right)$ persist when parameter $\epsilon$ is finite, the next question is whether the manifolds associated with a single point $v_{0} \in \mathcal{M}_{0}$ also perturb in a similar fashion for finite $\epsilon$. Since 

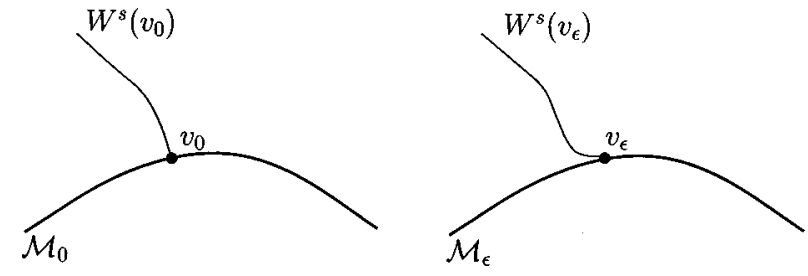

FIG. 2. A sketch of $W^{s}\left(v_{0}\right)$ and $W^{s}\left(v_{\epsilon}\right)$.

individual points $v_{\epsilon} \in \mathcal{M}_{\epsilon}$ are no longer fixed points, it is not clear that these structures will remain after the perturbation of $\epsilon$.

It turns out that these structures will still remain, although the dynamic nature of the system in this region is quite different from in the case $\epsilon=0$. A sketch of the stable manifolds $W^{s}\left(v_{0}\right)$ and $W^{s}\left(v_{\epsilon}\right)$ is given in Fig. 2.

The existence of these structure is shown by determining a graph function which describes the manifold associated with a single given point on $\mathcal{M}_{\epsilon}$.

Theorem 3.2 [Fenichel (1979)]: If $\epsilon>0$ is sufficiently small, then

(1) in $a_{2}=0$ [which is $W^{s}\left(\mathcal{M}_{\epsilon}\right)$ ] there exists, for each $v_{\epsilon}$ $=(\hat{x}, \epsilon) \in \mathcal{M}_{\epsilon}$, a function $x=h_{s}^{v}(b)$ defined for $|b| \leqslant \Delta$ such that the graphs

$$
W^{s}\left(v_{\epsilon}\right)=\left\{(x, 0, b, \epsilon): x=h_{s}^{v}(b)\right\}
$$

form a locally invariant family;

(2) in $b_{2}=0$ [which is $W^{u}\left(\mathcal{M}_{\epsilon}\right)$ ] there exists, for each $v_{\epsilon}$ $=(\hat{x}, \epsilon) \in \mathcal{M}_{\epsilon}$, a function $x=h_{u}^{v}(a)$ defined for $|a| \leqslant \Delta$ such that the graphs

$$
W^{u}\left(v_{\epsilon}\right)=\left\{(x, a, 0, \epsilon): x=h_{u}^{v}(a)\right\}
$$

\section{form a locally invariant family.}

These graphs, $h_{s}^{v}(b)$ and $h_{u}^{v}(a)$, define the stable and unstable manifolds associated with a given point $v_{\epsilon} \in \mathcal{M}_{\epsilon}$. Individual points on these manifolds are known as fibers, and will approach the trajectory on $\mathcal{M}_{\epsilon}$ that passes through the basepoint of the fiber. Hence, the fibers "tie together" the fast and slow dynamics in a precise way. Results for how points in $W^{s}\left(\mathcal{M}_{\epsilon}\right)$ decay to $\mathcal{M}_{\epsilon}$ are given below where the flow generated by the vector field is defined by $\phi_{t}(\cdot)$ and the evolution of $u$ under that flow is given by $\phi_{t}(u)$.

Corollary 1 [Fenichel (1979)]. $\kappa_{s}>0$ and $\alpha_{s}<0$ so that if $u \in W^{s}(v)$ then

$$
\left|\phi_{t}(u)-\phi_{t}(v)\right| \leqslant \kappa_{s} e^{\alpha_{s} t}
$$

for all $t \geqslant 0$ under the assumption that the solution does not leave the neighborhood of the manifold $\mathcal{M}_{\epsilon}$. Furthermore, similar results exist for the unstable manifold.

The graphs, $h_{s}^{v}(b)$ and $h_{u}^{v}(a)$, define mappings from $\left(v_{\epsilon}, b\right)$ to $\left(h_{s}^{v}(b), b\right)$ or from $\left(v_{\epsilon}, a\right)$ to $\left(h_{u}^{v}(a), a\right)$, respectively. By taking the inverse of these mapping, the base points of the fibers which are on $\mathcal{M}_{\epsilon}$ can be found. This inverse mapping takes a fiber in $W^{s}\left(\mathcal{M}_{\epsilon}\right)$ or $W^{u}\left(\mathcal{M}_{\epsilon}\right)$ to its base on $\mathcal{M}_{\epsilon}$.
These inverses can be defined as $\pi^{-}:(x, b, \epsilon) \rightarrow \hat{x} \in \mathcal{M}_{\epsilon}$ and $\pi^{+}:(x, a, \epsilon) \rightarrow \hat{x} \in \mathcal{M}_{\epsilon}$. By using these inverses as a coordinate transformation, the fibers inside $W^{s}\left(\mathcal{M}_{\epsilon}\right)$ and $W^{u}\left(\mathcal{M}_{\epsilon}\right)$ can also be straightened. This is done first by straightening the stable manifold

$$
\begin{aligned}
& x_{3}=\pi^{-}\left(x_{2}, b_{2}, \epsilon\right), \\
& a_{3}=a_{2}, \\
& b_{3}=b_{2},
\end{aligned}
$$

and then by straightening the fibers of the unstable manifold,

$$
\begin{aligned}
& x_{4}=\pi^{+}\left(x_{3}, a_{3}, \epsilon\right), \\
& a_{4}=a_{3}, \\
& b_{4}=b_{3} .
\end{aligned}
$$

By making these transformations, the slow flow becomes decoupled from the flow on the manifolds $W^{s}\left(\mathcal{M}_{\epsilon}\right)$ and $W^{u}\left(\mathcal{M}_{\epsilon}\right)$. If either $a=0$ or $b=0$ then what was $f(x, a, b, \epsilon)$ is only a function of $x$ and $\epsilon$ in these new coordinates. For this reason, the new form of the equations in these transformed coordinates is given by

$$
\begin{aligned}
& \dot{x}_{4}=\epsilon\left\{h\left(x_{4}, \epsilon\right)+H\left(x_{4}, a_{4}, b_{4}, \epsilon\right)\right\}, \\
& \dot{a}_{4}=\Lambda\left(x_{4}, a_{4}, b_{4}, \epsilon\right) a_{4}, \\
& \dot{b}_{4}=\Gamma\left(x_{4}, a_{4}, b_{4}, \epsilon\right) b_{4},
\end{aligned}
$$

where $H\left(x_{4}, a_{4}, b_{4}, \epsilon\right)$ is a bilinear function of $a_{4}$ and $b_{4}$. This change of coordinates will be valid only in a local neighborhood of the manifold, which can be defined as the set $D=\left\{\left(x_{4}, a_{4}, b_{4}, \epsilon\right):\left|a_{4}\right| \leqslant \Delta,\left|b_{4}\right| \leqslant \Delta, x_{4} \in K, \epsilon \in\left[0, \epsilon_{0}\right]\right\}$. This is the Fenichel normal form which is derived in Jones, Kaper, and Kopell (1996). In the proofs that will follow, the Fenichel normal form [Eq. (34)] will be used but the subscripts will be omitted.

\section{THEORETICAL RESULTS}

In this section, a justification for the slow manifold identification algorithm of Maas and Pope will be given for a system of ODEs in the standard singular perturbation form. For the limiting case where the time separation between the slow and fast dynamics is infinite $(\epsilon \rightarrow 0)$, the proof is fairly straightforward. In the case where $\epsilon$ is small but finite, the Fenichel normal form is utilized to show that the Maas and Pope algorithm identifies the proper slow manifold. For the results presented here, it will be assumed that the dynamics appear in the standard form for singularly perturbed systems. When the dynamics is not in this form originally, there always exists a nonlinear change of coordinates to put them in this form (Fenichel, 1979). For this reason, working with the standard form of singular perturbed systems is not problematic.

\section{A. Infinite time-scale separation}

For the limiting case $\epsilon \rightarrow 0$, the representation of the dynamics with the fast time scaling (4) will be utilized. To prove that the Maas and Pope algorithm identifies the slow 
manifold correctly in this limiting case, first it will be shown that the condition described in the Maas and Pope algorithm is satisfied on the slow manifold.

Theorem 4.1: For the system described by Eq. (4) under the assumptions given in Sec. III, $Q_{L}^{T}(z) F(z)=0$ for $z$ $\in \mathcal{M}_{0}$.

Proof: For this set of equations, the dynamics can be linearized about a point $\left(x, h^{0}(x)\right) \in \mathcal{M}_{0}$. The linearization of Eq. (4) takes the form

$$
\left(\begin{array}{l}
\dot{\delta} x \\
\dot{\delta} y
\end{array}\right)=\left(\begin{array}{cc}
0 & 0 \\
0 & \frac{\partial g}{\partial y}\left(x, h^{0}(x), 0\right)
\end{array}\right)\left(\begin{array}{l}
\delta x \\
\delta y
\end{array}\right)
$$

on $\mathcal{M}_{0}$. This is because $(\partial g / \partial x)\left(x, h^{0}(x), 0\right)=0$ and follows from

$\frac{d g}{d x}\left(x, h^{0}(x), 0\right)=\frac{\partial g}{\partial x}\left(x, h^{0}(x), 0\right)+\frac{\partial g}{\partial y}\left(x, h^{0}(x), 0\right) \frac{\partial h^{0}}{\partial x}(x)$

$$
=0 .
$$

The eigenvalues of the Jacobian given in (35) can be decomposed into two distinct groups. The first group of eigenvalues is identically zero and the set of eigenvectors associated with these eigenvalues is contained in the space defined by the orthonormal vectors $\left\{\left(\begin{array}{c}\epsilon_{1} \\ 0\end{array}\right),\left(\begin{array}{c}\epsilon_{2} \\ 0\end{array}\right), \ldots,\left(\begin{array}{l}\epsilon_{1} \\ 0\end{array}\right)\right\}$ which form a basis for the space $\mathfrak{R}^{l}$. Since the matrix $(\partial g / \partial y)\left(x, h^{0}(x), 0\right)$ is nonsingular, the eigenvalues of this matrix are bounded away from zero. The invariant eigenvector space associated with these nonzero eigenvalues is

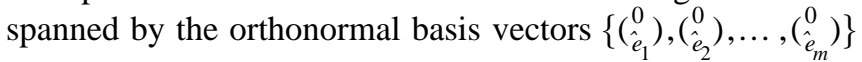
which span the invariant space, which will be called $\mathcal{E}$. These basis vectors should span the same invariant space as the rows of $Q_{L}^{T}$ as both sets of vectors correspond to eigenvalues which are nonzero.

In mathematical terms, this projection onto the invariant space associated with the nonzero eigenvalues is given by

$$
\left(\begin{array}{cc}
0 & \hat{e}_{1}^{T} \\
0 & \hat{e}_{2}^{T} \\
0 & \vdots \\
0 & \hat{e}_{m}^{T}
\end{array}\right)\left(\begin{array}{c}
\boldsymbol{\epsilon}\left(x, h^{0}(x), 0\right) \\
g\left(x, h^{0}(x), 0\right)
\end{array}\right)=0,
$$

as $g\left(x, h^{0}(x), 0\right)=0$ by the definition of $h^{0}(x)$. On the invariant manifold $\mathcal{M}_{0}$, the projection of the original dynamics onto the space $\mathcal{E}$ is identically zero, as can be seen from Eq. (38). Therefore, $Q_{L}^{T} F\left(x_{0}, y_{0}\right)=0$ on $\mathcal{M}_{0}$ and the conditions specified in the Maas and Pope (1992) algorithm are satisfied.

For points $\left(x_{0}, y_{0}\right)$ not on $\mathcal{M}_{0}$, it can be shown that the Maas and Pope algorithm will not find the quantity $Q_{L}^{T} F\left(x_{0}, y_{0}\right)$ is zero. If it is possible for this quantity to be zero for $\left(x_{0}, y_{0}\right) \notin \mathcal{M}_{0},\left(x_{0}, y_{0}\right)$ might be improperly classified as being on $\mathcal{M}_{0}$ by the algorithm.

Theorem 4.2: For the system described by Eq. (4) under the assumptions given in Sec. III, $Q_{L}^{T}(z) F(z) \neq 0$ for $z \notin \mathcal{M}_{0}$.

Proof: The linearization of (4) about $\left(x_{0}, y_{0}\right) \notin \mathcal{M}_{0}$ takes the following form:

$$
\left(\begin{array}{l}
\dot{\delta} x \\
\dot{\delta} y
\end{array}\right)=\left(\begin{array}{cc}
0 & 0 \\
\frac{\partial g}{\partial x}\left(x_{0}, y_{0}, 0\right) & \frac{\partial g}{\partial y}\left(x_{0}, y_{0}, 0\right)
\end{array}\right)\left(\begin{array}{c}
\delta x \\
\delta y
\end{array}\right) .
$$

As long as $(\partial g / \partial y)\left(x_{0}, y_{0}, 0\right)$ is nonsingular, this matrix has exactly $l$ eigenvalues which are zero and the invariant space associated with these eigenvalues is spanned by the basis vectors

$$
\left\{\left(\begin{array}{c}
e_{1} \\
0
\end{array}\right),\left(\begin{array}{c}
e_{2} \\
0
\end{array}\right), \ldots,\left(\begin{array}{c}
e_{l} \\
0
\end{array}\right)\right\}
$$

The orthogonal complement to this space (that space which refers to nonzero eigenvalues) will be spanned by the basis vectors

$$
\left\{\left(\begin{array}{c}
0 \\
\hat{e}_{1}
\end{array}\right),\left(\begin{array}{c}
0 \\
\hat{e}_{2}
\end{array}\right), \ldots,\left(\begin{array}{c}
0 \\
\hat{e}_{m}
\end{array}\right)\right\}
$$

Therefore, off the manifold the expression $Q_{L}^{T} F\left(x_{0}, y_{0}\right)$ is nonzero since for $\left(x_{0}, y_{0}\right) \notin \mathcal{M}_{0}, g\left(x_{0}, y_{0}, 0\right) \neq 0$ by definition, and

$$
\left(\begin{array}{cc}
0 & \hat{e}_{1}^{T} \\
0 & \hat{e}_{2}^{T} \\
0 & \vdots \\
0 & \hat{e}_{m}^{T}
\end{array}\right)\left(\begin{array}{c}
\epsilon f\left(x_{0}, y_{0}, 0\right) \\
g\left(x_{0}, y_{0}, 0\right)
\end{array}\right) \neq 0 .
$$

\section{B. Finite time-scale separation}

The slow manifold in the case where the time-scale separation is finite is $\mathcal{M}_{\epsilon}$. This manifold may not be the same as $\mathcal{M}_{0}$, so it should be shown that the algorithm proposed by Maas and Pope also properly identifies $\mathcal{M}_{\epsilon}$ from the equations given in (3). In order to show this rigorously, the Fenichel Normal Form will be used. Working with the coordinates in the Fenichel Normal Form, it can be shown that the algorithm of Maas and Pope properly identifies the slow manifold.

Theorem 4.3: For the system described by Eq. (3) under the assumptions given in Sec. III, $Q_{L}^{T}(z) F(z)=0$ for $z$ $\in \mathcal{M}_{\epsilon}$.

Proof: The Fenichel normal form will be used to prove this statement. Since the transformation to the normal form is smooth and invertible, the algorithm of Maas and Pope can be validated in these coordinates. First, Eq. (34) is linearized about a point $(x, 0,0) \in \mathcal{M}_{\epsilon}$.

$$
\begin{aligned}
& \dot{\delta} x=\epsilon \frac{\partial h}{\partial x}(x, \epsilon) \delta x, \\
& \dot{\delta} a=\Lambda(x, 0,0, \epsilon) \delta a, \\
& \dot{\delta} b=\Gamma(x, 0,0, \epsilon) \delta b .
\end{aligned}
$$

The Jacobian of the linearization is 


$$
J_{\epsilon}=\left(\begin{array}{ccc}
\epsilon \frac{\partial h}{\partial x}(x, \epsilon) & 0 & 0 \\
0 & \Lambda(x, 0,0, \epsilon) & 0 \\
0 & 0 & \Gamma(x, 0,0, \epsilon)
\end{array}\right),
$$

and the eigenvalues of $J_{\epsilon}$ lie in two distinct groups. The first group of eigenvalues consists of $m$ eigenvalues which have a real part with absolute value of magnitude $\mathcal{O}(\epsilon)$. The eigenvectors associated with these eigenvalues are contained in the space spanned by the vectors

$$
\left\{\left(\begin{array}{c}
e_{1} \\
0 \\
0
\end{array}\right),\left(\begin{array}{c}
e_{2} \\
0 \\
0
\end{array}\right), \ldots,\left(\begin{array}{c}
e_{l} \\
0 \\
0
\end{array}\right)\right\}
$$

where the vectors $e_{i}$ form a basis of the space $\mathfrak{R}^{l}$. Those eigenvalues which have real part which is bounded away from the origin have eigenvectors contained in the space spanned by the vectors

$$
\left\{\left(\begin{array}{c}
0 \\
\hat{e}_{1} \\
0
\end{array}\right), \ldots\left(\begin{array}{c}
0 \\
\hat{e}_{m_{a}} \\
0
\end{array}\right),\left(\begin{array}{c}
0 \\
0 \\
\check{e}_{1}
\end{array}\right) \ldots,\left(\begin{array}{c}
0 \\
0 \\
\check{e}_{m_{b}}
\end{array}\right)\right\} .
$$

The dynamics of system (34) evaluated for $(x, 0,0) \in \mathcal{M}_{\epsilon}$ is described by

$$
\dot{x}=\epsilon h(x, \epsilon), \quad \dot{a}=0, \quad \dot{b}=0 .
$$

Once again it is easily seen that the projection of the dynamics onto the space of the fast eigenvalues is identically zero on the manifold.

$$
\left(\begin{array}{ccc}
0 & \hat{e}_{1}^{T} & 0 \\
& \vdots & \\
0 & \hat{e}_{m_{a}}^{T} & 0 \\
0 & 0 & \check{e}_{1}^{T} \\
& \vdots & \\
0 & 0 & \check{e}_{m_{b}}^{T}
\end{array}\right)\left(\begin{array}{c}
\epsilon h(x, \epsilon) \\
0 \\
0
\end{array}\right)=0 .
$$

Since the projection of the dynamics onto the eigenvectors associated with the fast direction is identically zero, the Maas and Pope algorithm should again give the proper result when on the manifold $\mathcal{M}_{\epsilon}$.

For points not on the manifold $\mathcal{M}_{\epsilon}$, it is conjectured that the Maas and Pope criteria will not be satisfied. However, because the Fenichel normal form only holds in a local neighborhood of the slow manifold it is difficult to prove this fact rigorously. While the authors are able to show that the Maas and Pope criteria will not be satisfied on the manifolds $W^{s}\left(\mathcal{M}_{\epsilon}\right)$ and $W^{u}\left(\mathcal{M}_{\epsilon}\right)$ in the neighborhood of the slow manifold, extending these results to the more general case of points off the manifold is more difficult and an elegant method of proving this conjecture has not yet been found.

\section{EXAMPLE: BINARY DISTILLATION}

Distillation is used widely throughout the chemical and petroleum industries for separation and purification of products. A distillation column consists of a series of trays where vapor-liquid equilibrium of the mixture is achieved. In this manner, the product stream at the top of the column consists of the lighter components of the mixture and the product stream at the bottom of the column consists of the heavier components. There has been a great deal of work directed at understanding the dynamics and improving control of distillation processes because of its importance in these industries.

Modeling of the process in a tray-by-tray fashion is straightforward by considering component balances. In the simplest case of a binary mixture with $100 \%$ tray efficiency, constant molar flow, and constant molar holdups, tray $i$ is modeled as

$$
M_{i} \dot{x}_{i}=L x_{i-1}+V y_{i+1}-L x_{i}-V y_{i},
$$

where $x_{i}$ is the liquid composition on tray $i, y_{i}$ is the vapor composition on tray $i, L$ is the liquid molar flow rate, $V$ is the vapor flow rate, and $M_{i}$ is the molar holdup of tray $i$. This is the relationship that describes the change in molar holdup with respect to the flows onto and off of the tray. By assuming the vapor liquid equilibrium is governed by constant relative volatility $(\alpha), y_{i}$ is given by

$$
y_{i}=k\left(x_{i}\right)=\frac{\alpha x_{i}}{\left(1+(\alpha-1) x_{i}\right)} .
$$

This set of differential algebraic equations can be easily integrated, and early computational studies of the transient behavior of distillation columns are presented by Rosenbrock (1957).

The overall mathematical model of the distillation column is given by a number of differential equations that is equal to the number of trays of the column. For example, a column with $N$ trays and a pure liquid feed is described by

$$
\begin{aligned}
& M \dot{x}_{1}=V y_{2}-L_{u} x_{1}-D x_{1}, \\
& M \dot{x}_{2}=L_{u} x_{1}+V y_{3}-L_{u} x_{2}-V y_{2}, \\
& \vdots, \\
& M \dot{x}_{f-1}=L_{u} x_{f-2}+V y_{f}-L_{u} x_{f-1}-V y_{f-1}, \\
& M \dot{x}_{f}=L_{u} x_{f-1}+V y_{f+1}-L_{u} x_{f}-V y_{f}+F x_{f}, \\
& M \dot{x}_{f+1}=L_{l} x_{f}+V y_{f+2}-L_{l} x_{f+1}-V y_{f+1}, \\
& \vdots, \\
& M \dot{x}_{N-1}=L_{l} x_{N-1}+V y_{N+1}-L_{l} x_{N}-V y_{N}, \\
& M \dot{x}_{N}=L_{l} x_{N-1}-B x_{N}-V y_{N},
\end{aligned}
$$

where $D=V-L$ is the distillate flow rate, $B=L+F-V$ is the bottoms flow rate, $F$ is the feed flow rate, and $x_{f}$ is the feed composition. In order for the amount of material in the column to be constant, the liquid flows in the column above the feed $\left(L_{u}\right)$ and below the feed $\left(L_{l}\right)$ are related by $L_{l}$ $=L_{u}+F$. In industry, the goal is often to maintain a constant 
purity of either one or both of the product streams. The product stream at the top of the column is known as the distillate $\left(x_{d}=x_{1}\right)$ and the product stream at the bottom is known as the bottoms $\left(x_{b}=x_{N}\right)$. Control is performed by adjusting the flows of the column $(L, V, D, B)$ such that the overall mass balance in the column is conserved.

While these detailed models have been available for a long time, there have been numerous attempts to quantify the dynamics behavior of these differential algebraic equations and to develop a reduced-order model which accurately describes the dynamics. It has been well-known for a long time that the dynamics of a distillation column is dominated by a single large time constant which can be estimated by considering the column as a giant "mixing tank" (Davidson, 1956; Moczek, Otto, and Williams, 1965; Wahl and Harriot, 1970; Skogestad and Morari, 1987). Later, the multiple input/ multiple output dynamics of the linearized system were studied and a second smaller time constant was discovered which has important implications for control purposes (Skogestad and Morari, 1988). Input directionality is important when determining this second time constant, and these ideas are extended in the work of Sagfors and Waller (1995). Other recent approaches to this problem utilize nonlinear methods. Nonlinear wave theory is utilized in the work of Hwang (1991) and Hwang (1995). A singular perturbation theory approach, which will be described later in this section, is applied by Lévine and Rouchon (1991).

The example which will be studied is a binary distillation column with 40 ideal trays, a reboiler, and a total condenser described by Eq. (47). Constant molar overflow and constant relative volatility $(\alpha=1.5)$ are assumed. The feed is liquid with a light component composition $z_{f}=0.5$. The liquid molar holdup of each tray is assumed to be equal and is defined as $M_{i}=M=0.5$. The distillate flow rate is $D / F$ $=0.5$ and the liquid reflux flow rate is $L / F=2.702$ at the operating point of interest. The composition of the distillate output for these operating point is $x_{d}=x_{1}=0.99$. This column is identical to Column A from Skogestad and Morari (1988)

In order to study the transient dynamics around the operating point, the system is initialized at another steady state where $L / F=9.0$ and $D / F=0.2$. Since the reflux $(L / D)$ is higher at this operating point, the distillate has a higher purity $\left(x_{d}=0.999\right)$. At time $t=0$, the flows of the distillate and liquid reflux are changed to those of the operating point of interest $(L / F=2.702, D / F=0.5)$. From the simulation results of the dynamic transition to the steady state, the behavior appears to show evidence of a time-scale separation. The response of the distillate, after the first 50 minute transient period, appears to be an output from a first-order system (Fig. 3). To further illustrate the dynamics of the simulated system, a snapshot of the composition profile along the length of the column is given in Fig. 4. The composition profile is given at times $[0,50,100,200,300,400,500,600]$. As time progresses, the composition moves from the initial high purity profile to lower purity (as time passes, the profiles move toward the lower right hand corner). Since the output seems to suggest that the system exhibits behavior consistent with a one-dimensional slow manifold, three methods will be

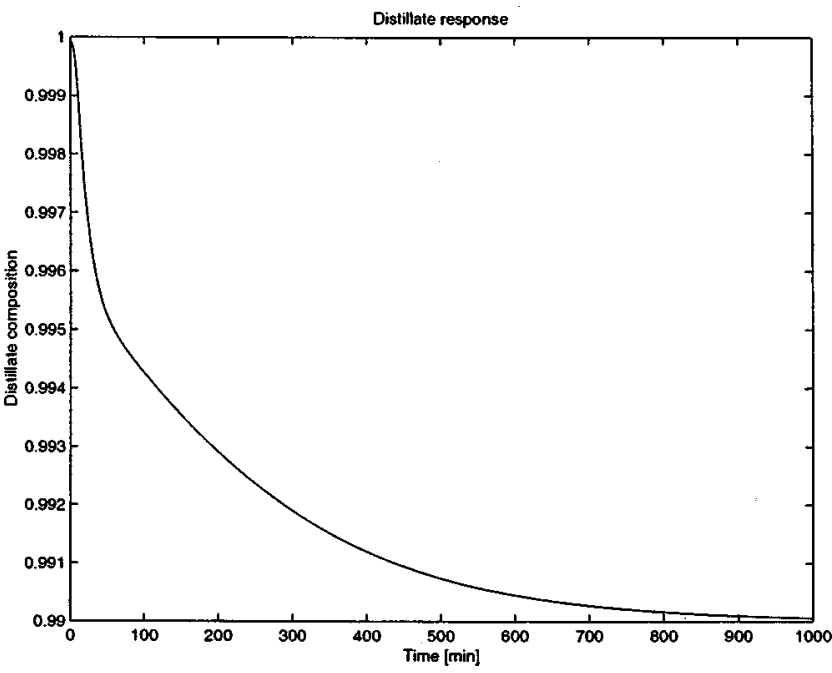

FIG. 3. Time response of the distillate composition for $L / F: 9.0 \rightarrow 2.702$, $D / F: 0.2 \rightarrow 0.5$ at $t=0$.

presented for identifying the one-dimensional "slow" manifold and the results will be compared with the simulation results.

\section{A. Linear modal analysis about a fixed point}

In the first method, modal decomposition will be applied to a linearized model of the column dynamics. A linear model of the system can be built at the operating point of Column A $(L / F=2.702, D / F=0.5)$. Consider Eq. (47) written in the form

$$
\dot{x}=f(x, u), \quad y=x_{1} .
$$

In this case, the inputs of interest $u$ are $L$ and $D$. The linearization about a given operating point $\left(x_{0}\right)$ can be described in deviation variables by

$$
\dot{q}=A q+B u, \quad z=[1,0, \ldots, 0] q,
$$

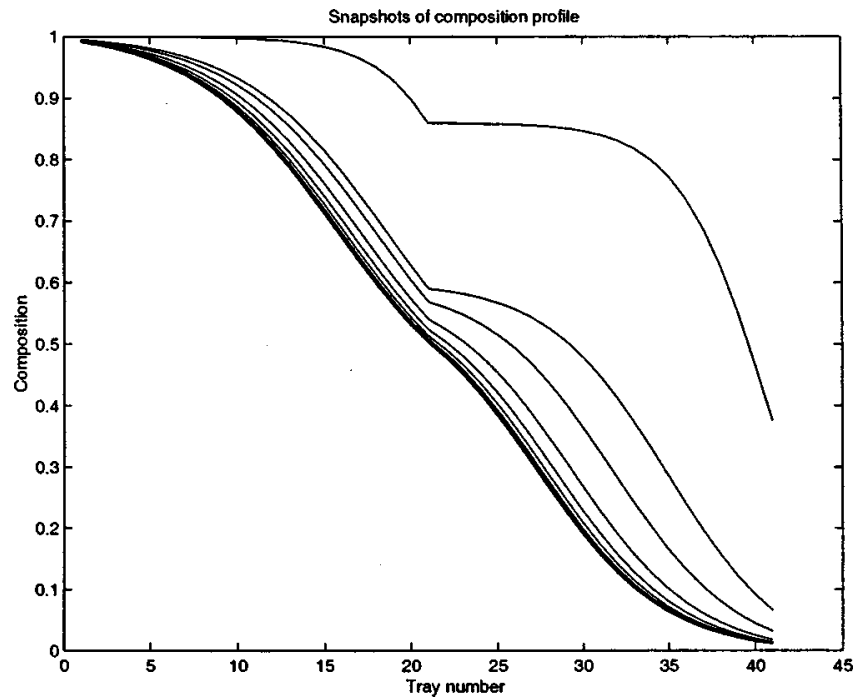

FIG. 4. Snapshots of the column composition profile at times $[0,50,100,200,300,400,500,600]$. 


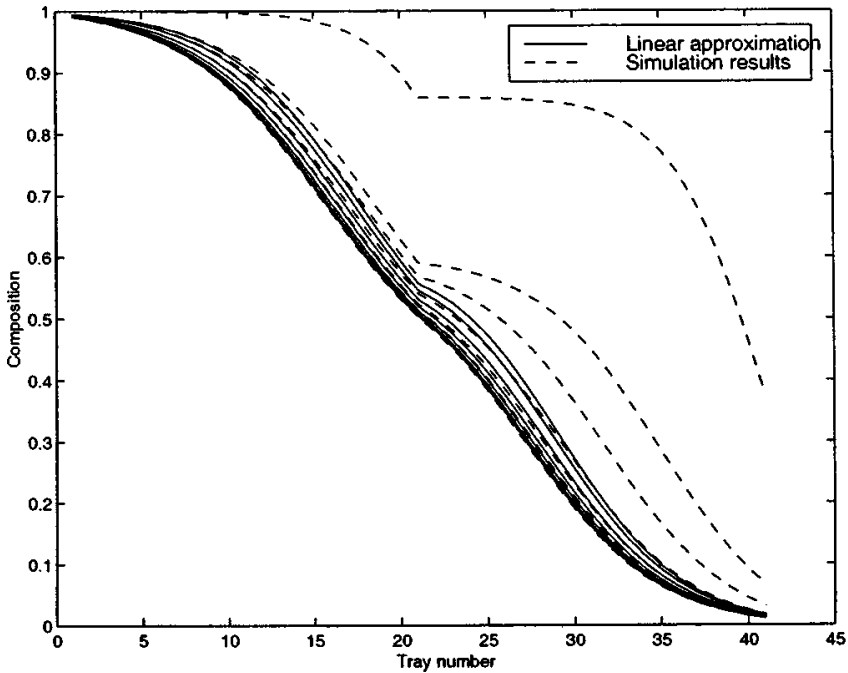

FIG. 5. The linear approximation to the one-dimensional slow manifold at times $[50,100,200,300,400,500,600]$.

where $A=(\partial f / \partial x)\left(x_{0}\right), B=(\partial f / \partial x)\left(x_{0}\right)$, and the output matrix takes this form since the output of interest is the distillate $x_{1}$. The variables $q, u$, and $z$ of this system are deviation variables about the steady state. By performing a modal decomposition and reducing the system such that only a single mode is kept, the following model is found:

$$
\dot{\bar{x}}_{1}=\lambda_{1} \bar{x}_{1}+B_{T}^{\star} u, \quad y=[1,0, \ldots, 0] t_{1} \bar{x}_{1},
$$

where $\lambda_{1}$ is the eigenvalue of smallest magnitude and $T$ $=\left[t_{1}, t_{2}, \cdots, t_{41}\right]$ is a matrix consisting of the eigenvectors $t_{i}$ of $A$ sorted such that $t_{1}$ is the eigenvector associated with the eigenvalue $\lambda_{1}$ (for ideal binary distillation, all the eigenvalues of $A$ are negative real and distinct). In the neighborhood of the operating point, system (50) should be a good approximation to the dynamics of the full system since $\left|\lambda_{1}\right|$ is more than an order of magnitude smaller than the other eigenvalues.

From the above reduced model,

$$
y=t_{11} \bar{x}_{1},
$$

where $t_{11}$ is the first component of the eigenvector associated with the dominant eigenvalue. By assuming the remaining modes are at equilibrium $\left(\bar{x}_{2}=\cdots=\bar{x}_{41}=0\right)$, the original state $x$ can be found by the transformation

$$
t_{1} \bar{x}_{1}=x \text {. }
$$

Using Eqs. (51) and (52), the state profile from the onedimensional reduced model can be found from the output $y$

$$
x=\frac{y}{t_{11}} t_{1} .
$$

This method is used to determine the linear approximation of the one-dimensional slow manifold of the distillation system. As can be seen in Fig. 5, this approximation does a good job of finding the low-dimensional manifold when the column profile is close to the steady state. This is expected, since a linear approximation is valid in a small neighborhood of the steady state. However, for short times after the step change, this method is unable to capture the features of the composition profile because the system is far from its steady state. This is due to the fact that the modal decomposition method only utilizes a linear model which is only valid in a local neighbor of the final steady state.

\section{B. A singular perturbation analysis}

In an attempt to find a better approximation of the slow manifold, nonlinear methods will be used. The second method which will be described here involves transforming the original equations describing the distillation column into the standard form for singularly perturbed systems (system 1). This physically motivated transformation is given in Lévine and Rouchon (1991) and will be used here to compute the slow manifold associated with this assumption.

The dynamic description of a section of $N$ trays of a distillation column with no feed is

$$
\begin{aligned}
& M \dot{x}_{1}=L x_{0}+V y_{2}-L x_{1}-V y_{1}, \\
& \vdots \\
& M \dot{x}_{j-1}=L x_{j-2}+V y_{j}-L x_{j-1}-V y_{j-1}, \\
& M \dot{x}_{j}=L x_{j-1}+V y_{j+1}-L x_{j}-V y_{j}, \\
& M \dot{x}_{j+1}=L x_{j}+V y_{j+2}-L x_{j+1}-V y_{j+1} \vdots \\
& M \dot{x}_{N}=L x_{N-1}+V y_{N+1}-L x_{N}-V y_{N} .
\end{aligned}
$$

To put the original system equations in the standard form of singularly perturbed systems, the following change of coordinates is made (Lévine and Rouchon, 1991):

$$
\left(\begin{array}{c}
x_{1} \\
\vdots \\
x_{j-1} \\
x_{j} \\
x_{j+1} \\
\vdots \\
x_{N}
\end{array}\right) \rightarrow\left(\begin{array}{c}
x_{1}^{f}=x_{1} \\
\vdots \\
x_{j-1}^{f}=x_{j-1} \\
x^{s}=\sum_{i=1}^{N} \frac{x_{i}}{N} \\
x_{j+1}^{f}=x_{j+1} \\
\vdots \\
x_{N}^{f}=x_{N}
\end{array}\right),
$$

where $x^{s}$ corresponds to the slow state, $x^{f}$ corresponds to fast states, and the section has a total of $N$ trays.

By defining the molar holdup of the entire section (the total number of moles in a given section) as $\bar{M}=N M$, this transformation results in the following description:

$$
\begin{aligned}
& \frac{1}{N} \bar{M} \dot{x}_{1}^{f}=L x_{0}+V y_{2}^{f}-L x_{1}^{f}-V y_{1}^{f}, \\
& \vdots \\
& \frac{1}{N} \bar{M} \dot{x}_{j-1}^{f}=L x_{j-2}^{f}+V k\left(x^{s}-\frac{1}{N} \sum_{i \neq j} x_{i}^{f}\right)-L x_{j-1}^{f}-V y_{j-1}^{f}, \\
& \bar{M} \dot{x} s=L x_{0}+V y_{N+1}-L x_{N}^{f}-V y_{1}^{f},
\end{aligned}
$$




$$
\begin{aligned}
& \frac{1}{N} \bar{M} \dot{x}_{j+1}^{f}=L\left(x^{s}-\frac{1}{N} \sum_{i \neq j} x_{i}^{f}\right)+V y_{j+2}^{f}-L x_{j+1}^{f}-V y_{j+1}^{f}, \\
& \vdots \\
& \frac{1}{N} \bar{M} \dot{x}_{N}^{f}=L x_{N-1}^{f}+V y_{N+1}-L x_{N}^{f}-V y_{N}^{f} .
\end{aligned}
$$

Note that these equations are in the standard form for a singularly perturbed system if the factor $1 / N$ is considered as the small parameter ( $\epsilon$ in the previous notation).

If it is assumed that the molar holdup of the entire column is large compared to the molar holdup on a single tray $(1 / N \rightarrow 0)$, the description of the slow system is given by

$$
\begin{aligned}
& 0=L x_{0}+V y_{2}^{f}-L x_{1}^{f}-V y_{1}^{f}, \\
& \vdots \\
& 0=L x_{j-2}^{f}+V y^{s}-L x_{j-1}^{f}-V y_{j-1}^{f}, \\
& \bar{M} \dot{x}^{s}=L x_{0}+V y_{N+1}-L x_{N}^{f}-V y_{1}^{f}, \\
& 0=L x^{s}+V y_{j+2}^{f}-L x_{j+1}^{f}-V y_{j+1}^{f}, \\
& \vdots \\
& 0=L x_{N-1}^{f}+V y_{N+1}-L x_{N}^{f}-V y_{N}^{f} .
\end{aligned}
$$

The reduced description of the dynamics of the system involves a single ODE with $N-1$ algebraic constraints.

In order to compute the slow manifold for Column $\mathrm{A}$, a term to account for the external feed $F x_{F}$ needs to be added to the right-hand side of the equation describing the feed tray. By defining the transformation such that $x_{j}$ is the feed tray, only the dynamic equation for $x^{s}$ will include terms resulting from the feed. There are also some slight modifications to the above equations because the first equation now describes the condenser $\left[x_{1}\right.$ of (47)] and the last equation describes the reboiler $\left[x_{N}\right.$ of (47)]. Applying the transformation of Eq. (55) to the equations describing Column A (47), the set of algebraic constraints describing the slow manifold are

$$
\begin{aligned}
& 0=V y_{2}^{f}-V x_{1}^{f}, \\
& 0=L x_{1}^{f}+V y_{3}^{f}-L x_{2}^{f}-V y_{2}^{f}, \\
& \vdots \\
& 0=L x_{j-2}^{f}+V y^{s}-L x_{j-1}^{f}-V y_{j-1}^{f}, \\
& 0=(L+F) x^{s}+V y_{j+2}^{f}-(L+F) x_{j+1}^{f}-V y_{j+1}^{f}, \\
& \vdots \\
& 0=(L+F) x_{N-2}^{f}+V y_{N}^{f}-(L+F) x_{N-1}^{f}-V y_{N-1}^{f}, \\
& 0=(L+F) x_{N-1}^{f}-(L+F-V) x_{N}^{f}-V y_{N}^{f} .
\end{aligned}
$$

The $N-1$ algebraic constraints defined above involve $N$ variables, so it is expected that the surface in the state-space which satisfies these algebraic conditions is a onedimensional manifold.

By specifying the distillate composition $x_{1}$, the remaining tray compositions can be found by solving Eq. (60). Us-

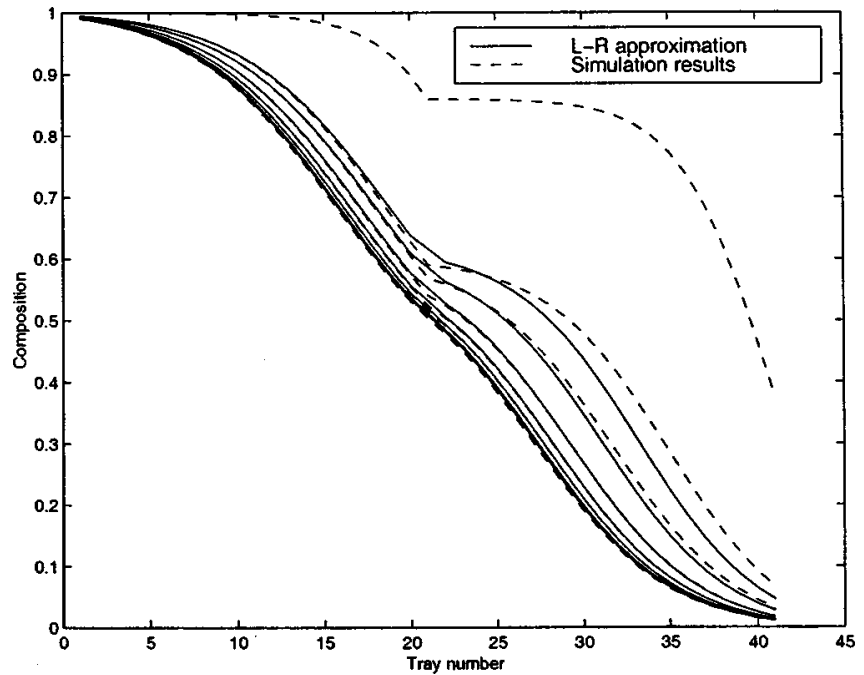

FIG. 6. The singular perturbation approximation of Lévin and Rouchon of the one-dimensional slow manifold at times [50,100,200,300,400,500,600].

ing the distillate composition from the simulation at the times corresponding to the snapshots, the slow manifold at this time can be found. The results of computing the slow manifold using this method are given in Fig. 6. It appears that this assumption captures the main characteristics of the profile.

\section{Determining the surface which satisfies the Maas-Pope criteria}

The computational algorithm of Maas and Pope (1992) is the third method applied to this example for computing the reduced manifold. The algorithm described in Sec. II is used to determine the slow manifold where $x_{1}$, the distillate composition, is used as a fixed parameter. The values of the distillate composition from the simulation at the times of interest are used as the fixed states for the minimization of (16). The NPSOL algorithm is used for computing the manifold, as described previously.

Computation of a single column profile on the slow manifold with a specified distillate composition takes approximately 10 minutes on a Sun SPARCstation20. For each of the eight profiles, the minimization ends up converging to zero. The results are given in Fig. 7. It appears that this computational algorithm of Maas and Pope (1992) does the best job of capturing the slow manifold of the three methods presented in terms of the error between the predicted profiles and simulation results of Maas and Pope.

In order to compare these methods, the norm of the difference between the simulation results and the profile given by the different methods of computing the slow manifold was found. The results presented are the two-norm of the difference between the profiles at the defined simulation times and can be found in Fig. 8. From this comparison, the Maas and Pope criteria have the smallest error in predicting the column profiles based on the assumptions and methods used in this section. 


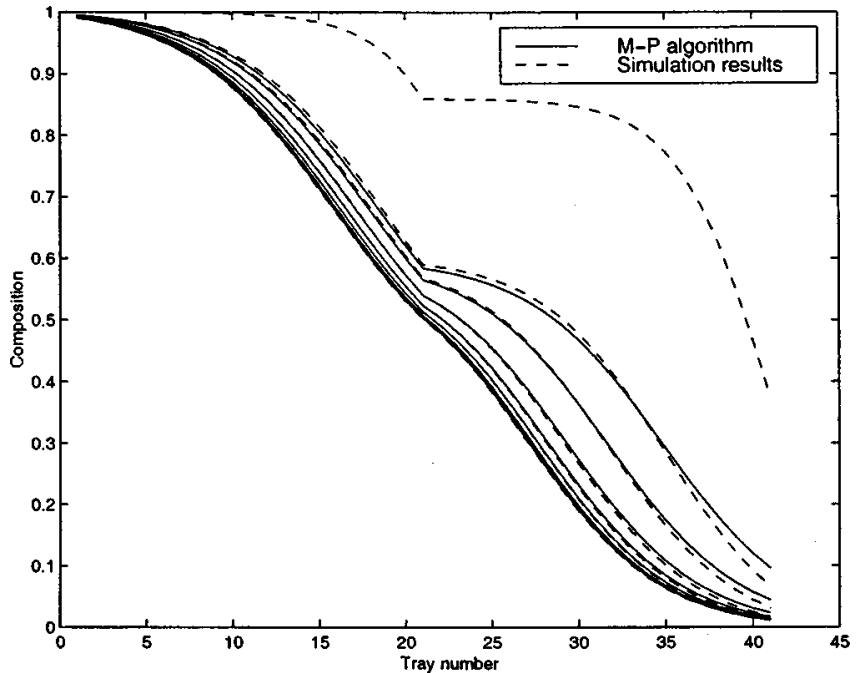

FIG. 7. The results of the Maas and Pope method of identifying the onedimensional slow manifold at times [50,100,200,300,400,500,600].

\section{PRELIMINARY THOUGHTS ON BUILDING REDUCED MODELS}

The Maas and Pope (1992) algorithm identifies the reduced manifold, so it should be possible to fit the equations that describe the manifold from the results of the algorithm. The output of the algorithm is a number of points on the $n_{r}$-dimensional slow manifold

$$
\begin{aligned}
& \left(z_{1}, z_{2}, \ldots, z_{n}\right)_{1}, \\
& \left(z_{1}, z_{2}, \ldots, z_{n}\right)_{2}, \\
& \vdots \\
& \left(z_{1}, z_{2}, \ldots, z_{n}\right)_{M} .
\end{aligned}
$$

The points on the slow manifold should satisfy $n-n_{r}$ nonlinear constraints.

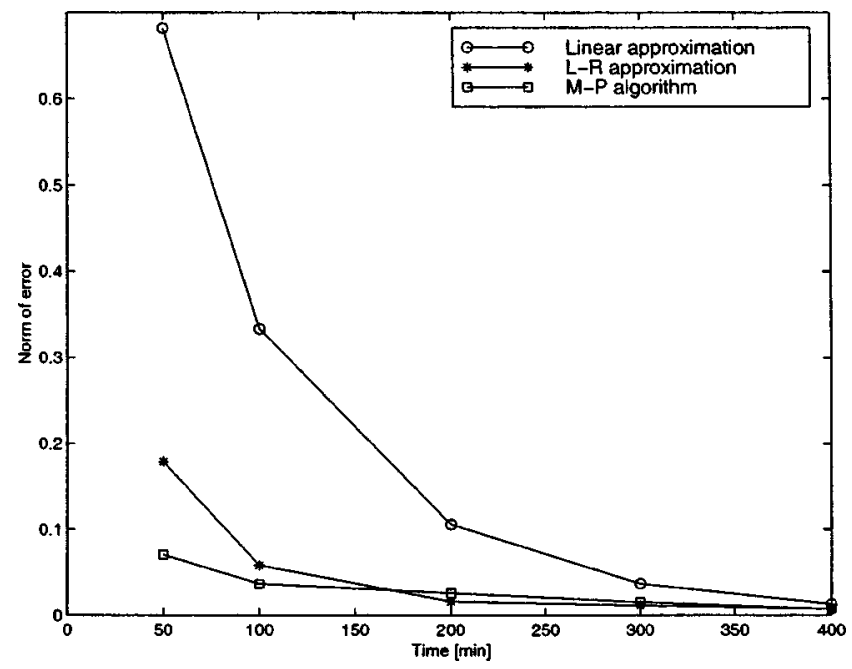

FIG. 8. The norm of the error associated with each approximation method as a function of time.
These nonlinear constraints can be estimated from the data by performing $n-n_{r}$ black-box identifications. The results of the black-box regressions can be defined as

$$
\begin{aligned}
& z_{n_{r}+1}=g_{1}\left(z_{1}, \ldots, z_{n_{r}}\right), \\
& z_{n_{r}+2}=G_{2}\left(z_{1}, \ldots, z_{n_{r}}\right), \\
& \vdots \\
& z_{n}=G_{n-n_{r}}\left(z_{1}, \ldots, z_{n_{r}}\right) .
\end{aligned}
$$

Once again these equations defining the constraints can be substituted into the original equations

$$
\begin{aligned}
& \dot{z}_{1}=F_{1}\left(z_{1}, \ldots, z_{n_{r}}, G_{1}(\mathbf{z}), \ldots, G_{n-n_{r}}(\mathbf{z})\right), \\
& \dot{z}_{2}=F_{2}\left(z_{1}, \ldots, z_{n_{r}}, G_{1}(\mathbf{z}), \ldots, G_{n-n_{r}}(\mathbf{z})\right), \\
& \vdots \\
& \dot{z}_{n_{r}}=F_{n_{r}}\left(z_{1}, \ldots, z_{n_{r}}, G_{1}(\mathbf{z}), \ldots, G_{n-n_{r}}(\mathbf{z})\right),
\end{aligned}
$$

where $\mathbf{z}=\left[z_{1}, \ldots, z_{n_{r}}\right]$. By making this substitution, a reduced model of the dynamics is formed.

While this reduced model will not have any fast dynamics if the functions $G_{i}$ are able to represent the slow manifold exactly, a black-box approximation may not exactly describe the manifold. If there is any mismatch between the manifold and the functions $G_{i}$, there may be mismatch between the slow dynamics of the original model and this reduced model, and there may also be a mismatch at steady state.

Another option, suggested by Maas and Pope (1992), would be to utilize a lookup table to determine the points on the reduced manifold. While this form may be more accurate than the black-box method described above, the reduced model could not be described by a set of differential equations. While a lookup table may be suitable for simulation, this form would probably not be compatible with existing methods of designing nonlinear controllers.

Possibly the best approach to determining a reduced model of the slow dynamics would be to use the Maas and Pope (1992) algorithm to determine an initial approximation of the slow manifold by fitting a function to the output of the algorithm. Note that the Maas and Pope algorithm will determine the slow manifold exactly, but the result is not an algebraic description. The fitted version of this manifold could then be used as a starting point for the algorithm of Roussel and Fraser (1991). This method is an iterative process which computes a reduced set of differential equations describing the reduced system. The iterative process of this algorithm is computationally expensive, so a good guess to the manifold is needed. To reduce the computational requirements of the algorithm of Roussel and Fraser (1991), the Maas and Pope algorithm could be utilized. There appears to be some initial work in this direction (Davis, 1997).

\section{EXAMPLE: TWO-PHASE CSTR}

In this example, the dynamics of a two-phase chemical reactor will be studied. The reactor has a pure gas feed consisting of chemical component $A$ and a pure liquid feed of 


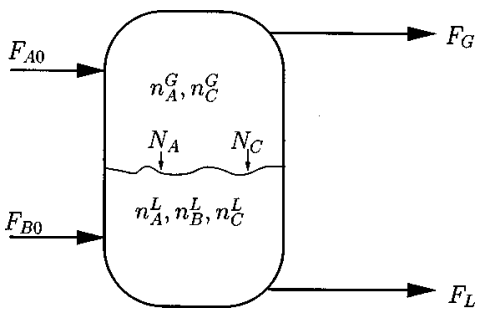

FIG. 9. Gas-liquid phase chemical reactor.

chemical component $B$. These react to form component $C$ $(A+B \rightarrow C)$. An illustration of the system is given in Fig. 9 . While components $A$ and $C$ exist in both the liquid and gas phase, component $B$ remains purely in the liquid phase. The effect of mass-transfer between the gas and liquid phase is also considered in the model. The equations describing the reactor are as follows:

$$
\begin{aligned}
& \frac{d n_{A}^{G}}{d t}=F_{A 0}-N_{A}-F_{G} y_{A}, \\
& \frac{d n_{C}^{G}}{d t}=-N_{C}-F_{G} y_{C}, \\
& \frac{d n_{A}^{L}}{d t}=N_{A}-k C_{A} C_{B} V_{L}-F_{L} x_{A}, \\
& \frac{d n_{B}^{L}}{d t}=F_{B 0}-k C_{A} C_{B} V_{L}-F_{L} x_{B}, \\
& \frac{d n_{C}^{L}}{d t}=N_{C}+k C_{A} C_{B} V_{L}-F_{L} x_{C},
\end{aligned}
$$

where $n_{i}$ is the number of moles of component $i$ in liquid (superscript $L$ ) or gas (superscript $G$ ) phase, $x_{i}$ is the mole fraction of liquid component $i, y_{i}$ is the mole fraction of gas component $i, V_{L}=\left(n_{A}^{L}+n_{B}^{L}+n_{C}^{L}\right) / \rho$ is the liquid volume, $C_{i}=n_{i}^{L} / V_{L}$ is the concentration in the liquid phase, $N_{i}$ $=k_{L}\left(x_{A}^{\star}-x_{A}\right)$ is the mass transfer from the gas to liquid phase of component $i, x_{i}^{\star}=P y_{i} / P_{i}^{\text {sat }}, P=\left(n_{A}^{G}+n_{C}^{G}\right) R T /$ $\left(V-V_{L}\right)$ is the reactor pressure, $k=k_{0} e^{-E_{a} / R T}$ is the reaction rate constant, with the parameters and variables at steady state given in Tables I and II.

Figures 10 and 11 give simulation results of the full dynamical system from arbitrary initial conditions $\left(\left[n_{A}^{G}, n_{C}^{G}, n_{A}^{L}, n_{B}^{L}, n_{C}^{L}\right]=[3000,1000,3300,10500,1500]\right)$. Two time scales appear to be present in the simulation results - an initial fast transient, and a slower time constant associated with the long time dynamics.

TABLE I. Reactor variables at steady-state.

\begin{tabular}{clc}
\hline \hline Variable & \multicolumn{1}{c}{ Description } & Steady-state value \\
\hline$n_{A}^{G}$ & Molar gas holdup of $A(\mathrm{~mol})$ & 3552 \\
$n_{C}^{G}$ & Molar gas holdup of $C(\mathrm{~mol})$ & 1238 \\
$n_{A}^{L}$ & Molar liquid holdup of $A(\mathrm{~mol})$ & 3061 \\
$n_{B}^{L}$ & Molar liquid holdup of $B(\mathrm{~mol})$ & 10630 \\
$n_{C}^{L}$ & Molar liquid holdup of $C(\mathrm{~mol})$ & 1310 \\
\hline \hline
\end{tabular}

TABLE II. Reactor parameters.

\begin{tabular}{lll}
\hline \hline Parameter & \multicolumn{1}{c}{ Description } & \multicolumn{1}{c}{ Value } \\
\hline$F_{A 0}$ & Inlet vapor flowrate $(\mathrm{mol} / \mathrm{s})$ & 175 \\
$F_{B 0}$ & Inlet liquid flowrate $(\mathrm{mol} / \mathrm{s})$ & 250 \\
$F_{G}$ & Outlet gas flowrate $(\mathrm{mol} / \mathrm{s})$ & 92.2 \\
$F_{L}$ & Outlet liquid flowrate $(\mathrm{mol} / \mathrm{s})$ & 284.2 \\
$R$ & Gas constant $(\mathrm{J} / \mathrm{mol} \mathrm{K})$ & 8.314 \\
$E_{a}$ & Activation energy $(\mathrm{J} / \mathrm{mol})$ & 110000 \\
$k_{0}$ & Preexponential reaction factor $\left(\mathrm{m}^{3} / \mathrm{mol} \mathrm{s}^{3}\right)$ & $10^{11}$ \\
$\rho$ & Molar liquid phase density $\left(\mathrm{mol} / \mathrm{m}^{3}\right)$ & 15000 \\
$V$ & Reactor volume $\left(\mathrm{m}^{3}\right)$ & 1.8 \\
$T$ & Reactor temperature $(\mathrm{K})$ & 341.5 \\
$k_{L}$ & Mass transfer coefficient $(\mathrm{mol} / \mathrm{s})$ & 2500 \\
$P_{A}^{\text {sat }}$ & Saturation vapor pressure for $A$ at $T(\mathrm{~Pa})$ & $51.11 \times 10^{6}$ \\
$P_{C}^{\text {sat }}$ & Saturation vapor pressure for $C$ at $T(\mathrm{~Pa})$ & $56.49 \times 10^{6}$ \\
\hline \hline
\end{tabular}

In Fig. 11 the first $200 \mathrm{~s}$ of the simulation are plotted, and the effect of the time-scale separation can be clearly seen. Some very fast dynamics occur in the first $20 \mathrm{~s}$. The behavior appears to be consistent with the mass-transfer of the system, since the liquid and gas holdups of components $A$ and $C$ (variables $n_{A}^{G}, n_{A}^{L}, n_{C}^{G}$, and $n_{C}^{L}$ ) adjust very quickly. After this initial transient, the behavior is consistent with a time scale of approximately $100 \mathrm{~s}$. Most likely, this behavior is associated with the chemical reaction.

\section{A. Perturbation analysis based on physical arguments}

One possible way to reduce this model is to use a physical understanding of the process as motivation. The assumption made here is that the mass-transfer dynamics is much faster than the other dynamics of the system. This means that the mass-transfer term reaches steady state much faster than the rest of the system. Therefore, in order to reduce the equations, it is assumed that $\dot{N}_{A}=\dot{N}_{C}=0$. This is equivalent to
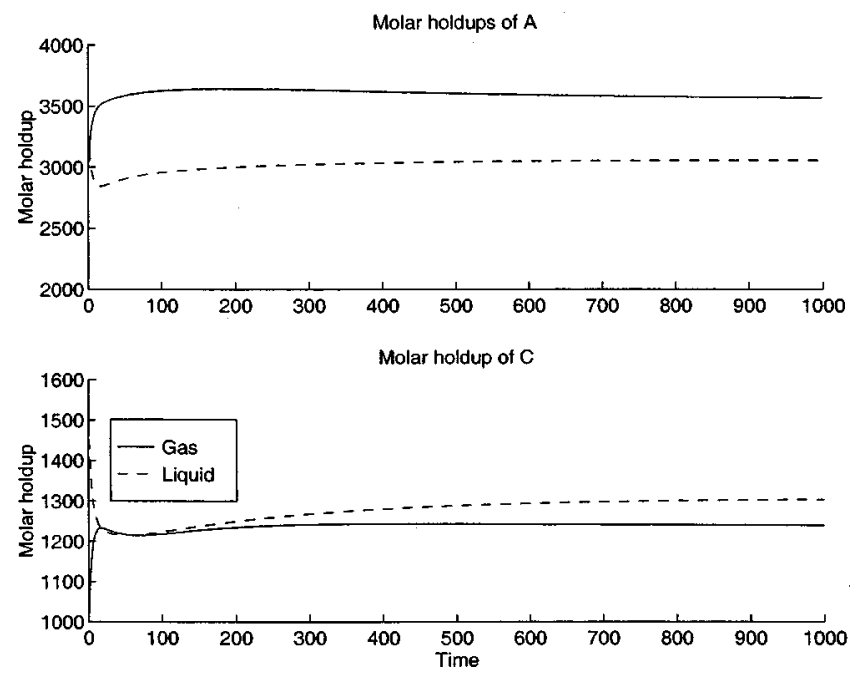

FIG. 10. Simulation of the full set of equations for the CSTR (top graph: $n_{A}^{G}$ - solid line, $n_{A}^{L}$-dashed line. Bottom graph: $n_{C}^{G}$-solid line, $n_{C}^{L}$-dashed line). 

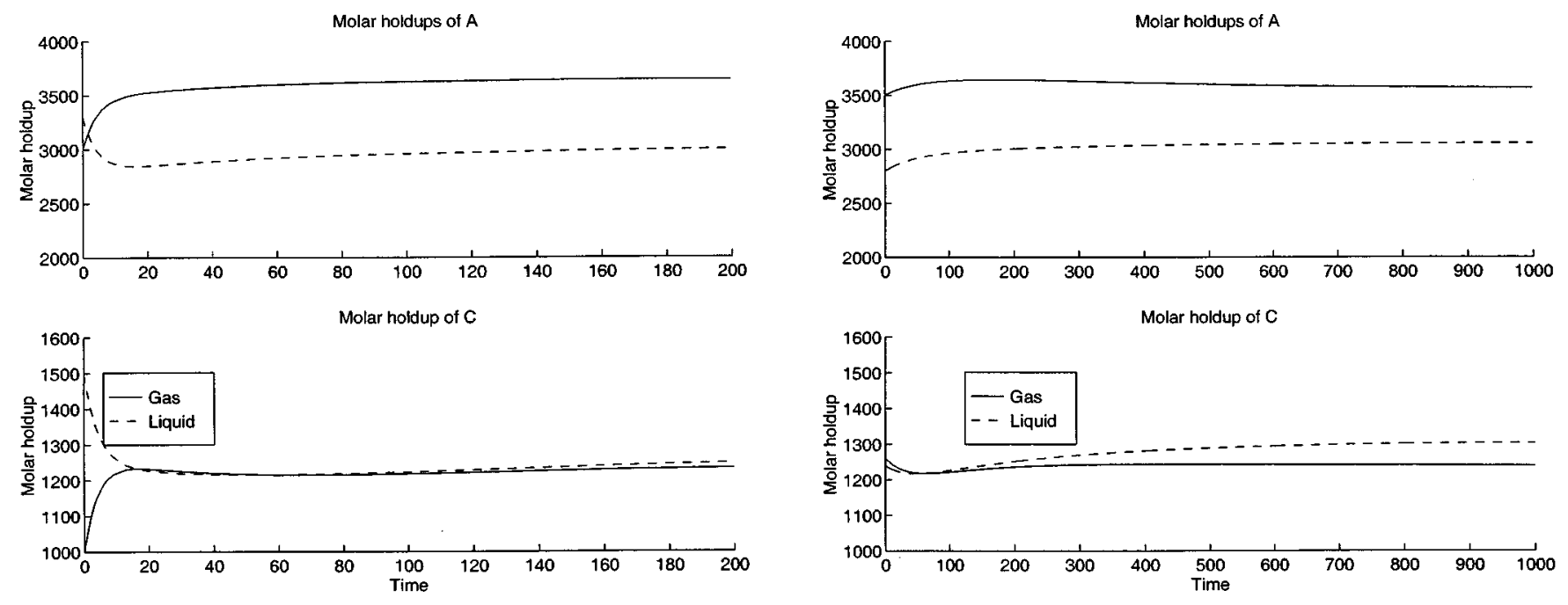

FIG. 11. Simulation of the full set of equations for the CSTR for the first 200 seconds.

forcing the mass-transfer dynamics to reach equilibrium infinitely fast and provides two algebraic constraints that describe the slow manifold.

By transforming to a new set of variables which describe the overall molar holdup of each of the individual components in the reactor $\left(\left[n_{A}=n_{A}^{G}+n_{A}^{L}, n_{B}^{L}, n_{C}=n_{C}^{G}+n_{C}^{L}\right]\right)$, the fast mass-transfer dynamics $\left(N_{A}\right.$ and $\left.N_{C}\right)$ will no longer appear. With these new coordinates, it is assumed that the important quantities for the slow dynamics are the total moles of $A$ and $C$ in the reactor. The new set of differential algebraic equations describing the reduced dynamics for the physically based model reduction scheme becomes

$$
\begin{aligned}
& \frac{d n_{A}}{d t}=F_{A 0}-F_{G} y_{A}-k C_{A} C_{B} V_{L}-F_{L} x_{A}, \\
& \frac{d n_{B}^{L}}{d t}=F_{B 0}-k C_{A} C_{B} V_{L}-F_{L} x_{B}, \\
& \frac{d n_{C}}{d t}=k C_{A} C_{B} V_{L}-F_{L} x_{C}-F_{G} y_{C} \\
& \left(\dot{x}_{A}^{\star}-\dot{x}_{A}\right)=0, \\
& \left(\dot{x}_{C}^{\star}-\dot{x}_{C}\right)=0 .
\end{aligned}
$$

It is possible to convert back to the original set of coordinates by utilizing the algebraic constraints for calculation.

Figures 12 and 13 give the results of simulation based on system (65). The initial conditions for this simulation are such that $\left[n_{A}, n_{B}^{L}, n_{B}\right]$ match the initial conditions of the full system. The concentration differences $n_{A}^{G}-n_{A}^{L}$ and $n_{C}^{G}-n_{C}^{L}$ are then adjusted until $\left(\dot{x}_{A}^{\star}-\dot{x}_{A}\right)=0$ and $\left(\dot{x}_{C}^{\star}-\dot{x}_{C}\right)=0$. This is the projection of the dynamics onto the slow manifold that results from the physically motivated model reduction scheme.

In Fig. 13 the fast dynamics do not appear. The reason is that the mass-transfer terms $N_{A}$ and $N_{C}$ do not appear in the differential equations. Notice that the long term dynamics is reproduced quite well using this approximation. However,

the simulated solution of this set of equations requires a combined differential algebraic equation solver. In addition, this model can only be used with control design schemes which are specifically developed for differential algebraic systems.

\section{B. Model reduction utilizing the Maas-Pope criteria}

The computational algorithm of Maas and Pope was then used to identify the three-dimensional manifold of slow dynamics. The variables $\left[n_{C}^{G}, n_{A}^{L}\right]$ are solved for as a function of the remaining variables $\left[n_{A}^{G}, n_{B}^{L}, n_{C}^{L}\right]$ over an equally spaced grid of 8000 points for molar holdups less than 300 mol from the steady state using the Maas and Pope algorithm. Computationally this takes approximately $15 \mathrm{~min}$ on a Sun SPARCstation 20. After this is completed, the variables $\left[n_{C}^{G}, n_{A}^{L}\right]$ are estimated as a function of the other variables using a quadratic polynomial. The fitted functions are
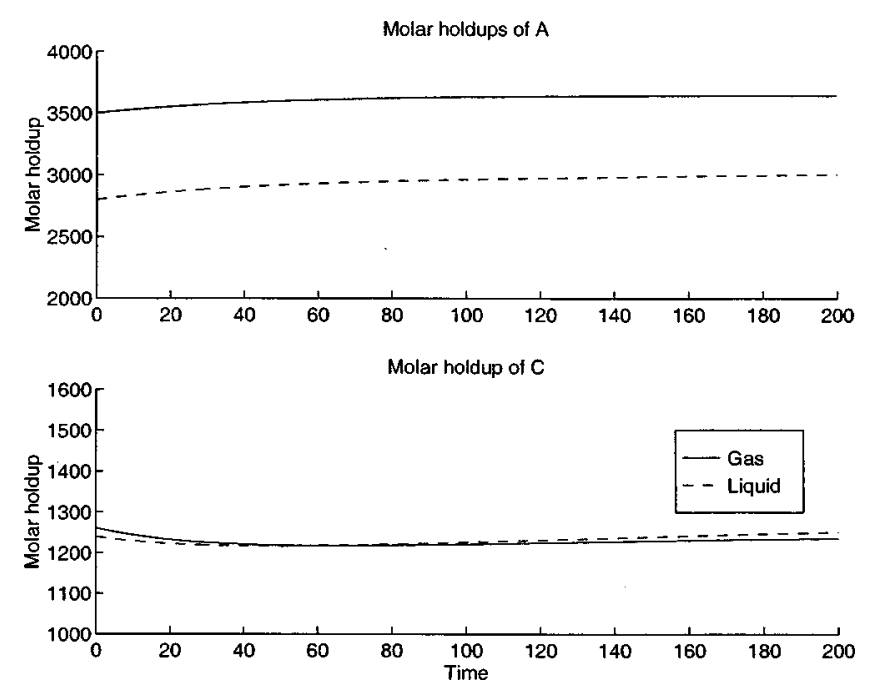

FIG. 13. Simulation results for the physically motivated model reduction in the first 200 seconds. 

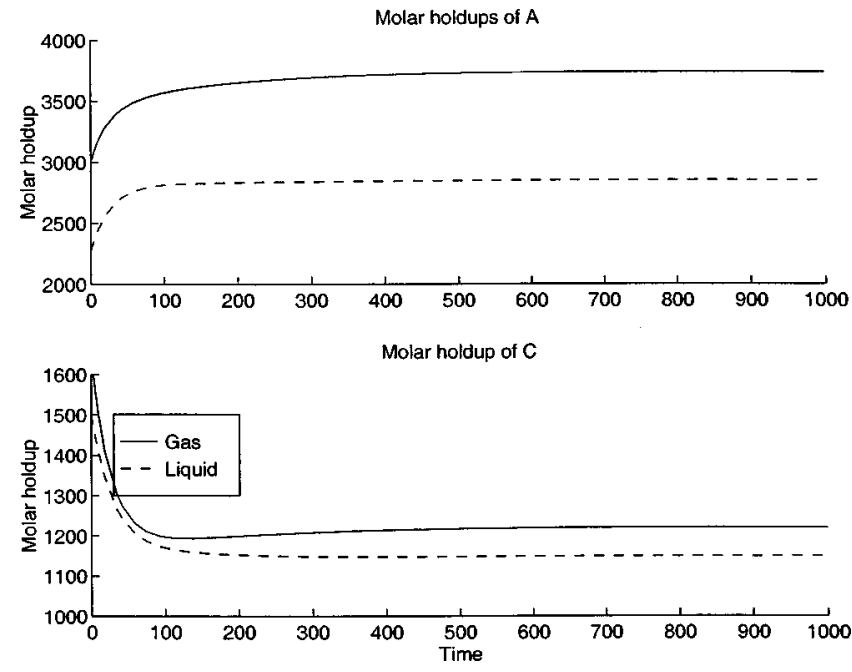

FIG. 14. Simulation results for the reduced model generated by black-box fitting of the results of the Maas and Pope algorithm.

$$
\begin{aligned}
& n_{C}^{G}=H_{1}\left(n_{A}^{G}, n_{B}^{L}, n_{C}^{L}\right), \\
& n_{A}^{L}=H_{2}\left(n_{A}^{G}, n_{B}^{L}, n_{C}^{L}\right) .
\end{aligned}
$$

Note that a tradeoff exists between the complexity of the fitted functions $H_{1}$ and $H_{2}$ and the accuracy of the model. The functions should describe the surface that satisfies the Maas and Pope criteria. However a high order description of $H_{1}$ and $H_{2}$ would lead to increased complexity of the reduced set of ODEs where the complexity refers to the number of terms appearing on the right-hand side of the reduced set of ODEs. This may not be desirable for control design procedures which rely on algebraic manipulations of the system to be controller. A quadratic function was used in order to develop a reduced model of reasonable complexity and accuracy.

Substituting these fitted functions into the original equations, reduced equations are as follows:

$$
\begin{aligned}
& \frac{d n_{A}^{G}}{d t}=F_{A 0}-N_{A}-F_{G} y_{A}, \\
& \frac{d n_{B}^{L}}{d t}=F_{B 0}-k C_{A} C_{B} V_{L}-F_{L} x_{B}, \\
& \frac{d n_{C}^{L}}{d t}=N_{C}+k C_{A} C_{B} V_{L}-F_{L} x_{C}, \\
& n_{C}^{G}=H_{1}\left(n_{A}^{G}, n_{B}^{L}, n_{C}^{L}\right), \\
& n_{A}^{L}=H_{2}\left(n_{A}^{G}, n_{B}^{L}, n_{C}^{L}\right) .
\end{aligned}
$$

Note that for this set of equations, the reduced form is more natural. By substituting the expressions $H_{1}\left(n_{A}^{G}, n_{B}^{L}, n_{C}^{L}\right)$ and $H_{2}\left(n_{A}^{G}, n_{B}^{L}, n_{C}^{L}\right)$ for $n_{C}^{G}$ and $n_{A}^{L}$, the system can easily be simulated as a set of only three differential equations. This is different than the system which results from the physically motivated model reduction where a differential algebraic solver is needed for simulation.

The results of the simulation of system (67) are given in Figs. 14 and 15. In these simulations, the initial values of
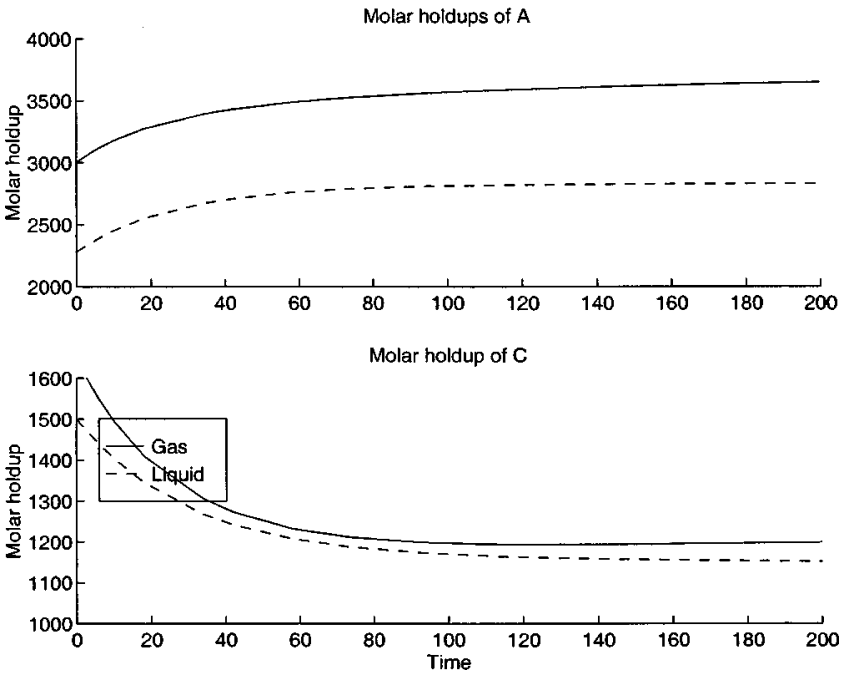

FIG. 15. Simulation results for the reduced model generated by black-box fitting of the results of the Maas and Pope algorithm for the first 200 seconds.

$\left[n_{A}^{G}, n_{B}^{L}, n_{C}^{L}\right]$ are specified to be identical to those values used in the simulation of the full system. The initial values of $\left[n_{C}^{G}, n_{A}^{L}\right]$ are determined from the constraints $H_{1}\left(n_{A}^{G}, n_{B}^{L}, n_{C}^{L}\right)$ and $H_{2}\left(n_{A}^{G}, n_{B}^{L}, n_{C}^{L}\right)$. Note that this choice of initial conditions for the reduced system is somewhat arbitrary. It is simply one projection of the initial conditions onto the slow manifold. The question of determining the optimal initial conditions on the slow manifold for an initial condition not in the neighborhood of the manifold is an open research question.

The simulations using this model no longer exhibit the fast dynamics of the full set of equations. In addition, it appears that the computed initial conditions for $\left[n_{C}^{G}, n_{A}^{L}\right]$ are similar to the full simulation result after the mass-transfer reaches steady state (around $20 \mathrm{~s}$ ). Dynamically, the results appear quite similar to the full simulation results if the initial transient is ignored. However, since the fit to the slow manifold is only approximate there is some error associated with the final steady state of the reduced model.

The cause of this steady-state error is due to the method by which $H_{1}$ and $H_{2}$ are determined. In this example, the functions have been fit such that the error is minimized over the entire portion of the slow manifold identified by the Maas and Pope algorithm. Another option would be to fit the constrained versions of the functions $H_{1}$ and $H_{2}$ such that the steady state error is zero. The effect would be to reduce the steady state error, but to decrease the accuracy by which the functions fit the results of the algorithm outside of the steady-state. A third option involves performing a weighted optimization such that errors in the region near the steadystate are penalized more than errors more distant.

The results of the quadratic fit and model reduction are a set of three differential equations which can be utilized easily by a nonlinear control scheme. Figure 16 compares the results of the three different models for the molar holdup of component $A$. 


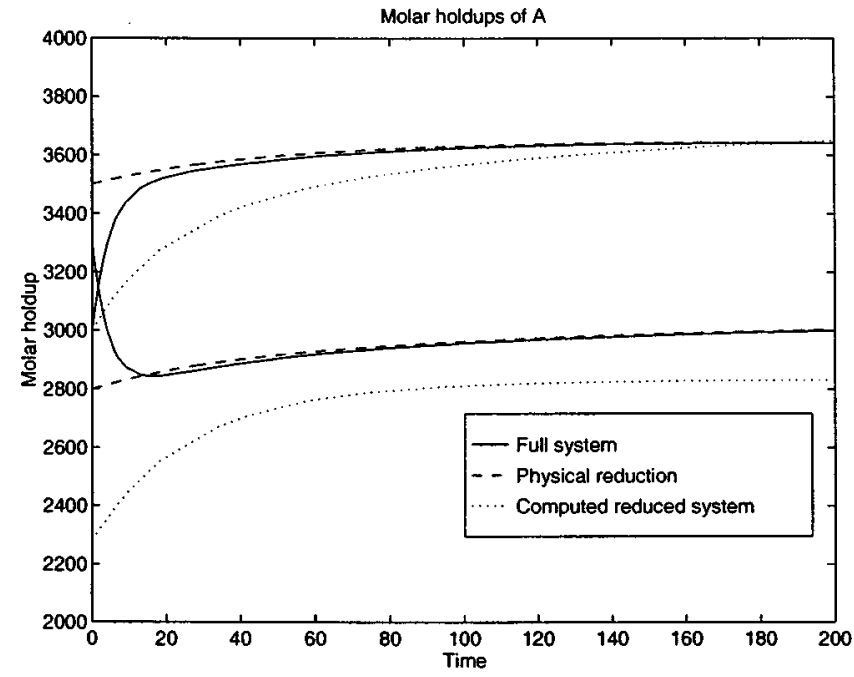

FIG. 16. Comparison of the three different models for the first 200 seconds.

\section{CONCLUSIONS}

The algorithm of Maas and Pope (1992) has been examined as a tool for nonlinear model reduction of systems which exhibit behavior with time-scale separations. First, it was shown that the algorithm identified the proper reduced manifold of slow dynamics for systems with infinite and finite time-scale separations. The slow manifold of a simulated binary distillation column was found using the algorithm and the results were compared with previously utilized methods of linear model reduction and physically based nonlinear model reduction. While the other methods make assumptions about the structure of the slow manifold, the Maas and Pope algorithm is able to exactly identify the intrinsic slow manifold of the system.

Some thoughts on how the results of the algorithm could be used for model reduction were then presented and this method of model reduction was used on a model of a twophase chemical reactor. The results were compared with the results of a standard physically based model reduction and the results of simulating the full system. In this example, the slow dynamics of the system was reproduced quite well by using the Maas and Pope algorithm and black-box modeling to arrive at a reduced system.

While it is shown that the Maas and Pope algorithm identifies the reduced manifold of slow dynamics, it is still not clear how to utilize this manifold in order to build a reduced model of the dynamics that is amenable to construction of a nonlinear controller. Possible work for the future also includes determining the optimal projection (if one exists) for arbitrary initial conditions onto the slow manifold.

Brogan, W. L. (1991). Modern Control Theory (Prentice-Hall, Englewood Cliffs, NJ).

Davidson, J. F. (1956). "The transient behavior of plate distillation columns," Trans. Inst. Chem. Eng. 34, 44-52.

Davis, M. J. (1997). Personal communication.

Fenichel, N. (1979). "Geometric singular perturbation theory for ordinary differential equations," J. Diff. Eqns. 31, 53-98.

Gill, P. E., Murray, W., Saunders, M. A., and Wright, M. H. (1994). User's guide for NPSOL 5.0: A FORTRAN package for nonlinear programming, Technical report, Stanford University.

Guillemin, V., and Pollack, A. (1974). Differential Topology (Prentice-Hall, Englewood Cliffs, NJ).

Horn, R. A., and Johnson, C. R. (1985). Matrix Analysis (Cambridge University Press, Cambridge).

Hwang, Y. (1991). "Nonlinear wave theory for dynamics of binary distillation columns," AIChE. J. 37, 705-723.

Hwang, Y. (1995). "Wave propagation in mass-transfer processes: From chromatography to distillation," Ind. Eng. Chem. Res. 34, 2849-2864.

Jones, C. (1994). "Geometric singular perturbation theory," in Dynamical Systems, No. 1609 in Lecture Notes in Mathematics, edited by R. Johnson (Springer, Montecatini Terme), pp. 45-118.

Jones, C., Kaper, T., and Kopell, N. (1996). "Tracking invariant manifolds up to exponentially small errors," SIAM (Soc. Ind. Appl. Math.) J. Math. Anal. 27, 558-577.

Khalil, H. K. (1996). Nonlinear Systems, 2nd ed. (Prentice-Hall, Upper Saddle River, NJ).

Kokotovic, P. V., and Khalil, H. K. (1986). Singular Perturbations in Systems and Control (IEEE, New York).

Lévine, J., and Rouchon, P. (1991). "Quality control of binary distillation columns via nonlinear aggregated models," Automatic 27, 463-480.

Maas, U., and Pope, S. B. (1992). "Simplifying chemical kinetics: Intrinsic low-dimensional manifolds in compositional space," Combust. Flame 88, 239-264.

Moczek, J. S., Otto, R. E., and Williams, T. J. (1965). “Approximation model for the dynamic response of large distillation columns," Chem. Eng. Prog. Symp. 61, 136-146.

Rosenbrock, H. H. (1957). "An investigation of the transient response of a distillation column Part I: Solution of the equations," Trans. Inst. Chem. Eng. 35, 234-351.

Roussel, M. R. and Fraser, S. J. (1991). "On the geometry of transient relaxation," J. Chem. Phys. 94, 7106-7113.

Sågfors, M. F. and Waller, K. V. (1995). "Dynamic low-order models for capturing directionality in nonideal distillation," Ind. Eng. Chem. Res. 34, 2038-2050.

Skogestad, S. and Morari, M. (1987). "The dominant time constant for distillation columns," Comput. Chem. Eng. 11, 607-617.

Skogestad, S. and Morari, M. (1988). "Understanding the dynamic behavior of distillation columns," Ind. Eng. Chem. Res. 27, 1848-1862.

Wahl, E. F. and Harriot, P. (1970). "Understanding and prediction of the dynamic behavior of distillation columns," Ind. Eng. Chem. Process Des. Dev. 9, 396-407. 